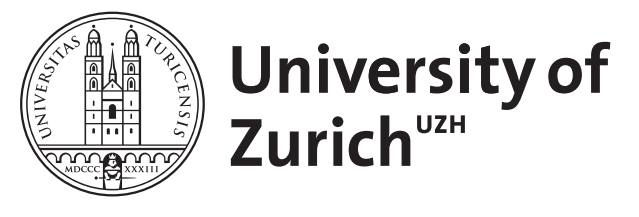

\title{
Quadri-tilings of the plane
}

de Tilière, $B$

\begin{abstract}
We introduce quadri-tilings and show that they are in bijection with dimer models on a family of graphs $\mathrm{R}^{*}$ arising from rhombus tilings. Using two height functions, we interpret a sub-family of all quadri-tilings, called triangular quadri-tilings, as an interface model in dimension $2+2$. Assigning "critical" weights to edges of $\mathrm{R}^{*}$, we prove an explicit expression, only depending on the local geometry of the graph $\mathrm{R}^{*}$, for the minimal free energy per fundamental domain Gibbs measure; this solves a conjecture of Kenyon (Invent Math 150:409-439, 2002). We also show that when edges of $\mathrm{R} *$ are asymptotically far apart, the probability of their occurrence only depends on this set of edges. Finally, we give an expression for a Gibbs measure on the set of all triangular quadri-tilings whose marginals are the above Gibbs measures, and conjecture it to be that of minimal free energy per fundamental domain.
\end{abstract}

DOI: https://doi.org/10.1007/s00440-006-0002-9

Posted at the Zurich Open Repository and Archive, University of Zurich ZORA URL: https://doi.org/10.5167/uzh-21581

Journal Article

Published Version

Originally published at:

de Tilière, B (2007). Quadri-tilings of the plane. Probability Theory and Related Fields, 137(3-4):487518.

DOI: https://doi.org/10.1007/s00440-006-0002-9 
Béatrice de Tilière

\title{
Quadri-tilings of the plane
}

Received: 15 March 2004 / Revised: 17 February 2006

Published online: 27 April 2006 - (C) Springer-Verlag 2006

\begin{abstract}
We introduce quadri-tilings and show that they are in bijection with dimer models on a family of graphs $\left\{R^{*}\right\}$ arising from rhombus tilings. Using two height functions, we interpret a sub-family of all quadri-tilings, called triangular quadri-tilings, as an interface model in dimension $2+2$. Assigning "critical" weights to edges of $R^{*}$, we prove an explicit expression, only depending on the local geometry of the graph $R^{*}$, for the minimal free energy per fundamental domain Gibbs measure; this solves a conjecture of Kenyon (Invent Math 150:409-439, 2002). We also show that when edges of $R^{*}$ are asymptotically far apart, the probability of their occurrence only depends on this set of edges. Finally, we give an expression for a Gibbs measure on the set of all triangular quadri-tilings whose marginals are the above Gibbs measures, and conjecture it to be that of minimal free energy per fundamental domain.
\end{abstract}

\section{Introduction}

In this paper, we introduce quadri-tilings and a sub-family of all quadri-tilings called triangular quadri-tilings. In order to explain the originality of this model, let us go back to the yet classical domino and lozenge tilings, see for example [3, $5,7,18]$. Both models are dimer models (see below) on a fixed graph, the square lattice $\mathbb{Z}^{2}$, and the equilateral triangular lattice $\mathbb{T}$, respectively. By the means of a height function, they can be interpreted as random discrete interfaces in dimension $2+1$, that is as random discrete surfaces of dimension 2 in a space of dimension 3 that have been projected to the plane [18]. Keeping this in mind, one can now explain the interesting feature of quadri-tilings: they correspond to dimer models on a family of graphs, instead of a fixed graph as was the case up to now. Moreover, by the means of two height functions, triangular quadri-tilings can be interpreted as random interfaces in dimension $2+2$, that is random surfaces of dimension 2 in a space of dimension 4 , that have been projected to the plane. It is the first such model arising from dimer models. Using tools of the dimer model, we are able to give an explicit expression for a Gibbs measure on triangular quadri-tilings, as well as a surprising property of the asymptotics of this measure. In the course of doing so, we prove a conjecture of [8].

Quadri-tilings are defined as follows. Consider the set of right triangles whose hypotenuses have length two. Color the vertex at the right angle black, and the other two vertices white. A quadri-tile is a quadrilateral obtained from two such triangles in two different ways: either glue them along the hypotenuse, or supposing they

B. de Tilière: Institut für Mathematik, Universität Zürich, Winterthurerstrasse 190, 8057 Zürich, Switzerland. e-mail: beatrice.detiliere@math.unizh.ch 
have a leg of the same length, glue them along this edge matching the black (white) vertex to the black (white) one, see Fig. 1. Note that both types of quadri-tiles have four vertices. A quadri-tiling of the plane is an edge-to-edge tiling of the plane by quadri-tiles that respects the coloring of the vertices, that is black (resp. white) vertices are matched to black (resp. white) ones. An example of quadri-tiling is given in Fig. 1. In all that follows, we consider quadri-tilings of the plane that use finitely many different quadri-tiles up to isometry.
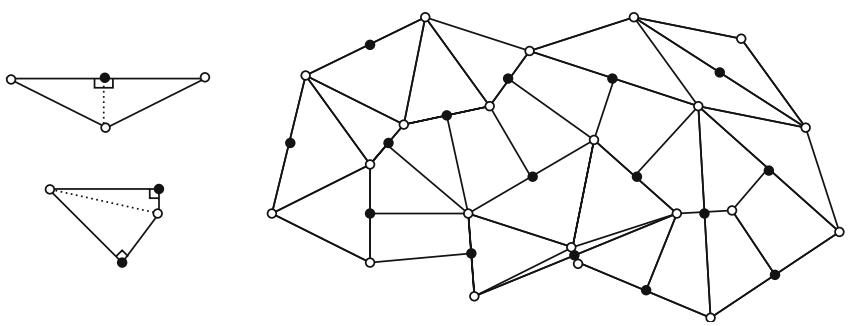

Fig. 1. Two types of quadri-tiles (left), and a quadri-tiling (right)

The goal of Sect. 2 is to precisely describe the features of quadri-tilings. In order to give some insight, let us define 2-tiling models or equivalently dimer models. A 2-tile of an infinite graph $G$ is a polygon made of two adjacent inner faces of $G$, and a 2-tiling of $G$ is a covering of $G$ with 2-tiles, such that there are no holes and no overlaps. The dual graph $G^{*}$ of $G$ is the graph whose vertices correspond to faces of $G$, two vertices of $G^{*}$ being joined by an edge if the corresponding faces are adjacent. A dimer configuration of $G^{*}$, also called perfect matching, is a subset of edges of $G^{*}$ which covers each vertex exactly once. Then 2-tilings of the graph $G$ are in bijection with dimer configurations of the dual graph $G^{*}$, as explained by the following correspondence. Denote by $f^{*}$ the dual vertex of a face $G$, and consider an edge $f^{*} g^{*}$ of $G^{*}$. We say that the 2-tile of $G$ made of the adjacent faces $f$ and $g$ is the 2-tile corresponding to the edge $f^{*} g^{*}$. Then, 2-tiles corresponding to edges of a dimer configuration of $G^{*}$ form a 2-tiling of $G$. Let us denote by $\mathcal{M}\left(G^{*}\right)$ the set of perfect matching of the graph $G^{*}$.

Prior to describing Sect. 2, we need one more definition. If $\mathrm{R}$ is a rhombus tiling of the plane, then the corresponding rhombus-with-diagonals tiling, denoted by $R$, is the graph obtained from $R$ by adding the diagonals of the rhombi. In the whole of this paper, we suppose that rhombus tilings of the plane have only finitely many rhombus angles.

In Sect. 2.1, we prove the main feature of quadri-tilings, i.e. that they correspond to 2-tilings on a family of graphs, which consists of rhombus-with-diagonals tilings. More precisely, if $T$ is a quadri-tiling, then by a geometric construction, we associate to $T$ a rhombus-with-diagonals tiling $R(T)$, such that $T$ is a 2-tiling of $R(T)$. The corresponding rhombus tiling $\mathrm{R}(T)$ is called the underlying rhombus tiling of $T$.

Triangular quadri-tilings consist in the sub-family of all quadri-tilings whose underlying tiling is a lozenge tiling, where lozenges are defined to be $60^{\circ}$-rhombi; refer to Fig. 2 for an example, and to Sect. 2.1 for the construction of the underlying 
tiling. In order to distinguish general rhombus tilings, denoted R, from lozenge tilings, we denote the latter by $L$. The set of all triangular quadri-tilings up to isometry is denoted by $\mathcal{Q}$. Note that $\mathcal{Q}$ corresponds to two superposed dimer models. Indeed, let $T$ be a triangular quadri-tiling, then $T$ is a 2-tiling of its underlying lozenge-with-diagonals tiling $L(T)$, moreover the corresponding lozenge tiling $\mathrm{L}(T)$ is a 2 -tiling of the equilateral triangular lattice $\mathbb{T}$.
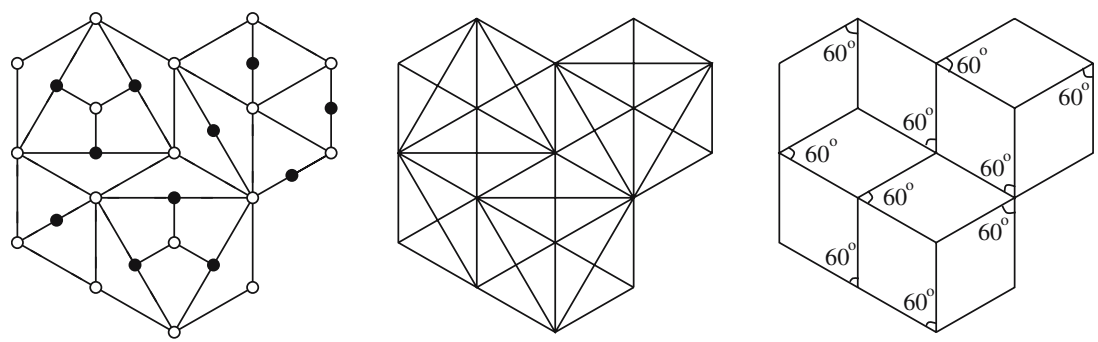

Fig. 2. Triangular quadri-tiling $T$ (left), underlying lozenge-with-diagonals tiling $L(T)$ (middle), corresponding lozenge tiling $\mathrm{L}(T)$ (right)

Section 2.2 consists in the geometric interpretation of triangular quadri-tilings using height functions. On the vertices of every triangular quadri-tiling $T$, we define a $\mathbb{Z}$-valued function $h_{1}$, called the first height function, corresponding to the "height" of $T$ interpreted as a 2-tiling of its underlying lozenge-with-diagonals tiling $L(T)$. Then, we assign a second height function $h_{2}$ (Thurston's height function on lozenges [18]) corresponding to the height of $L(T)$ interpreted as a 2-tiling of $\mathbb{T}$, see Fig. 4. Hence triangular quadri-tilings are characterized by two height functions, and so can be interpreted as discrete interfaces in dimension $2+2$. In Sect. 2.3, we give elementary operations that allow to transform any triangular quadri-tiling of a simply connected region into any other.

The dimer model belongs to the field of statistical mechanics, hence there are natural measures to consider, Boltzmann and Gibbs measures, which are defined as follows. Let $G$ be an infinite graph, and let $v$ be a positive weight function on the edges of $G^{*}$. Consider a finite sub-graph $G^{1}$ of $G$, then the Boltzmann measure on the set of dimer configurations $\mathcal{M}\left(G^{1 *}\right)$ of $G^{1^{*}}$, corresponding to the weight function $v$, is defined by

$$
\mu^{1}(M)=\frac{\prod_{e \in M} v(e)}{Z\left(G^{1^{*}}, v\right)},
$$

where $Z\left(G^{1^{*}}, v\right)=\sum_{M \in \mathcal{M}\left(G^{1 *}\right)} \prod_{e \in M} v(e)$ is the dimer partition function. A Gibbs measure is a probability measure on $\mathcal{M}\left(G^{*}\right)$ with the following property. If the matching in an annular region is fixed, the matchings inside and outside of the annulus are independent of each other, and the probability of any interior matching $M$ is proportional to $\prod_{e \in M} v(e)$.

An important question in solving a dimer model is the study of local statistics, i.e. to obtain an explicit expression for the set of Gibbs measures. Kenyon et al. [9] give such an expression for the two-parameter family of Gibbs measures 
on dimer configurations of doubly periodic bipartite graphs. The expression they obtain involves the limiting inverse Kasteleyn matrix which is hard to evaluate in general, often implying elliptic integrals. In another paper [8], for graphs $G$ which have bipartite duals and satisfy a geometric condition called isoradiality, Kenyon defines a specific weight function on the edges of $G^{*}$ called the critical weight function. He also defines the Dirac operator $K$ indexed by the vertices of $G^{*}$, and gives an explicit expression for its inverse $K^{-1}$ (see also Sects. 3.1 and 3.2). The expression for $K^{-1}$ has the interesting property of only depending on the local geometry of the graph. Kenyon conjectures that $K^{-1}$ is, in some sense to be determined, the limiting inverse Kasteleyn matrix. In Sect. 3, we consider a general rhombus-with-diagonals tiling of the plane $R$. It has the property of being an isoradial graph, so that we assign the critical weight function to edges of $R^{*}$. Theorem 4 of Sect. 3.3 (see also Theorem 1 below) proves an explicit expression for a Gibbs measure $\mu^{R}$ on $\mathcal{M}\left(R^{*}\right)$, as a function of $K$ and $K^{-1}$.

For every subset of edges $e_{1}=w_{1} b_{1}, \ldots, e_{k}=w_{k} b_{k}$ of $R^{*}$, the cylinder $\left\{e_{1}, \ldots, e_{k}\right\}$ is defined to be the set of dimer configurations of $R^{*}$ that contain these edges. Then

Theorem 1. There is a probability measure $\mu^{R}$ on $\mathcal{M}\left(R^{*}\right)$ such that, for every cylinders $\left\{e_{1}, \ldots, e_{k}\right\}$ of $R^{*}$,

$$
\mu^{R}\left(e_{1}, \ldots, e_{k}\right)=\left(\prod_{i=1}^{k} K\left(w_{i}, b_{i}\right)\right) \operatorname{det}_{1 \leq i, j \leq k}\left(K^{-1}\left(b_{i}, w_{j}\right)\right) .
$$

Moreover $\mu^{R}$ is a Gibbs measure on $\mathcal{M}\left(R^{*}\right)$. When $R^{*}$ is doubly periodic, $\mu^{R}$ is the unique Gibbs measure which has minimal free energy per fundamental domain among the two-parameter family of ergodic Gibbs measures of [9].

- Note that we do not ask the graph $R^{*}$ to be periodic. The proof of Theorem 1 is the subject of Sect. 4. The argument in the case where $R^{*}$ is not periodic relies on the argument in the case where $R^{*}$ is doubly periodic, combined with a non-trivial geometric property of rhombus tilings proved in Proposition 1: "every finite simply connected sub-graph of a rhombus tiling can be embedded in a periodic rhombus tiling of the plane."

- From the proof of Theorem 1, it appears that the statement is true for all doubly periodic isoradial graphs with bipartite duals. Hence, Theorem 1 solves the conjecture of [8] of interpreting the inverse Dirac operator as the limiting inverse Kasteleyn matrix. Moreover, the fact that the measure $\mu^{R}$ is of minimal free energy per fundamental domain makes it of special interest among the twoparameter family of ergodic Gibbs measures of [9].

- Using the locality property of $K^{-1}$ mentioned above, we deduce that the expression (1) only depends on the local geometry of the graph, hence it yields an easy way of computing local statistics explicitly. This is very surprising in regards of the expression obtained in [9], and we believe this locality property to be true only in the isoradial case with critical weights.

In Sect. 5, we extend the notion of Gibbs measure to the set of all triangular quadritilings $\mathcal{Q}$. Then, as a corollary to Theorem 1 we deduce an explicit expression for 
such a Gibbs measure $\mu$, and conjecture it to be that of minimal free energy per fundamental domain among a four parameter family of Gibbs measures.

In Sect. 6, we consider a general rhombus-with-diagonals tiling of the plane $R$. We assign the critical weight function to edges of $R^{*}$, and let $K$ be the Dirac operator indexed by vertices of $R^{*}$. Theorem 9 of Sect. 6.1 (see also Theorem 2 below) establishes that asymptotically (as $|b-w| \rightarrow \infty)$ and up to the second order term, $K^{-1}(b, w)$ only depends on the rhombi to which the vertices $b$ and $w$ belong, and else is independent of the structure of the graph $R$. For a general isoradial graph, Kenyon [8] gives an asymptotic formula for $K^{-1}(b, w)$ which depends on the angles of an edge-path from $w$ to $b$. Hence, it is an interesting and surprising fact that the dependence on the edges along the path should asymptotically disappear in the case of rhombus-with-diagonals tilings.

Theorem 2. As $|b-w| \rightarrow \infty, K^{-1}(b, w)$ is equal to

$$
\begin{aligned}
& \frac{1}{2 \pi}\left(\frac{1}{b-w}+\frac{\mathrm{e}^{-\mathrm{i}\left(\theta_{1}+\theta_{2}\right)}}{\bar{b}-\bar{w}}\right) \\
& \quad+\frac{1}{2 \pi}\left(\frac{\mathrm{e}^{2 \mathrm{i} \theta_{1}}+\mathrm{e}^{2 \mathrm{i} \theta_{2}}}{(b-w)^{3}}+\frac{\mathrm{e}^{-\mathrm{i}\left(3 \theta_{1}+\theta_{2}\right)}+\mathrm{e}^{-\mathrm{i}\left(\theta_{1}+3 \theta_{2}\right)}}{(\bar{b}-\bar{w})^{3}}\right)+O\left(\frac{1}{|b-w|^{3}}\right) .
\end{aligned}
$$

As a consequence of Theorem 2, we deduce that when edges $e_{1}, \ldots, e_{k}$ of $R^{*}$ are asymptotically far apart, $\mu^{R}\left(e_{1}, \ldots, e_{k}\right)$ only depends on the rhombi to which the edges $e_{1}, \ldots, e_{k}$ belong, and else is independent of the structure of the graph $R$ (Corollary 2). We conclude by giving a consequence of Corollary 2 for the measure $\mu$ on triangular quadri-tilings (Corollary 4 ).

\section{Features of quadri-tilings}

\subsection{Underlying rhombus-with-diagonals tilings}

Lemma 1. Quadri-tilings are in one-to-one correspondence with 2-tilings of graphs which are rhombus-with-diagonals tilings of the plane.

Proof. Consider a quadri-tiling of the plane $T$. Denote by $R$ the tiling of the plane obtained from $T$ by drawing, for each quadri-tile, the edge separating the two right triangles. Let $b$ be a black vertex of $R$, denote by $w_{1}, \ldots, w_{k}$ the neighbors of $b$ in cclw (counterclockwise) order. In each right triangle, the black vertex is adjacent to two white vertices, and since the gluing respects the coloring of the vertices, $w_{1}, \ldots, w_{k}$ are white vertices. Moreover, $b$ is at the right angle, so $k=4$ and the edges $w_{1} w_{2}, w_{2} w_{3}, w_{3} w_{4}, w_{4} w_{1}$ are hypotenuses of right triangles. Therefore $w_{1}, \ldots, w_{4}$ form a side-length-2 rhombus, and $b$ stands at the crossing of its diagonals. This is true for any black vertex $b$ of $R$, so $R$ is a rhombus-with-diagonals tiling of the plane, and $T$ is a 2-tiling of $R$.

As a consequence of Lemma 1, a quadri-tiling $T$ is a 2-tiling of a unique rhombuswith-diagonals tiling, which we call the underlying rhombus-with-diagonals tiling, and denote by $R(T)$, see Fig. 2 . 


\subsection{Height functions}

We define a first height function $h_{1}$ on vertices of every quadri-tiling $T$. Moreover, when $T$ is a triangular quadri-tiling, we define a second height function $h_{2}$ on vertices of $T$. Using $h_{1}$ and $h_{2}$, we interpret triangular quadri-tilings as discrete 2-dimensional surfaces in a 4-dimensional space projected to the plane.

\subsubsection{First height function}

Consider a quadri-tiling of the plane $T$, then $T$ is a 2-tiling of its underlying rhombus-with-diagonals tiling $R(T)$. In order to define the first height function $h_{1}$, we need a bipartite coloring of the faces of $R(T)$, which is given by the following.

Lemma 2. Let $\mathrm{R}$ be a rhombus tiling of the plane, and $R$ be the corresponding rhombus-with-diagonals tiling. Then $R$ has a bipartite coloring of its faces which is also a bipartite coloring of the vertices of $R^{*}$.

Proof. Cycles corresponding to the faces of the graph $\mathrm{R}$ have length four, thus $\mathrm{R}$ has a bipartite coloring of its vertices, say black and white. Consider a face of $R$ and orient its boundary edges cclw. If the white vertex of the hypotenuse-edge comes before the black one, assign color black to the face, else assign color white. This defines a bipartite coloring of the faces of $R$, which is also a bipartite coloring of the vertices of $R^{*}$ (see Fig. 3).

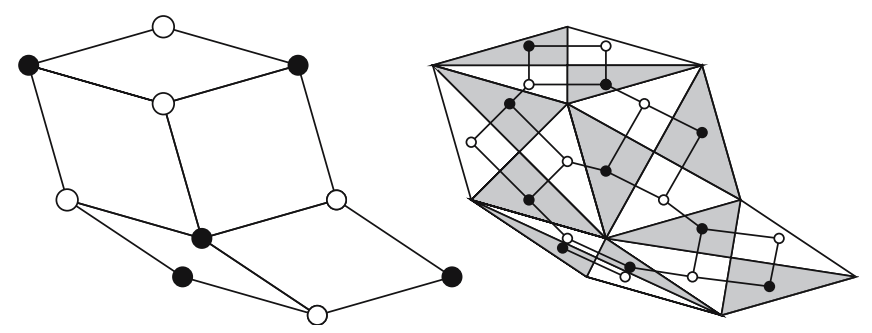

Fig. 3. Bipartite coloring of the vertices of R (left), and corresponding bipartite coloring of the faces of $R$ and of the vertices of $R^{*}$ (right)

Consider the bipartite coloring of the faces of $R(T)$. Orient the edges around the black faces cclw, edges around the white faces are then oriented cw, and define $h_{1}$ on the vertices of $T$ as follows. Fix a vertex $v_{1}$ on a boundary edge of a rhombus of $R(T)$, and set $h_{1}\left(v_{1}\right)=0$. For every other vertex $v$ of $T$, take an edge-path $\gamma_{1}$ from $v_{1}$ to $v$ which follows the boundaries of the quadri-tiles of $T$. The first height function $h_{1}$ changes by \pm 1 along each edge of $\gamma_{1}$ : if an edge is oriented in the direction of the path, then $h_{1}$ increases by 1 , if it is oriented in the opposite direction, then $h_{1}$ decreases by 1 . The value $h_{1}(v)$ is independent of the path $\gamma_{1}$ because the plane is simply connected, and the height change around any quadri-tile is zero. An example of computation of $h_{1}$ is given in Fig. 4 .

The following lemma gives a bijection between 2-tilings of a rhombus-withdiagonals tiling $R$ and first height functions defined on vertices of $R$. 
Lemma 3. Fix a vertex $v_{1}$ on a boundary edge of a rhombus of $R$. Let $\tilde{h}_{1}$ be a $\mathbb{Z}$-valued function on the vertices of $R$ satisfying the following two conditions:

- $\tilde{h_{1}}\left(v_{1}\right)=0$.

- $\tilde{h_{1}}(v)=\tilde{h_{1}}(u)+1$, or $\tilde{h_{1}}(v)=\tilde{h_{1}}(u)-2$, for any edge uv oriented from $u$ to $v$.

Then, there is a bijection between functions $\tilde{h}_{1}$ satisfying these two conditions and 2-tilings of $R$.

Proof. The idea of the proof closely follows [3]. If $T$ is a 2-tiling of $R$, then the first height function defined above satisfies the two conditions of the lemma: if an edge $u v$, oriented from $u$ to $v$, belongs to the boundary of a quadri-tile, it satisfies $h_{1}(v)=h_{1}(u)+1$, else if it lies across a quadri-tile, it satisfies $h_{1}(v)=h_{1}(u)-2$.

Conversely, consider a $\mathbb{Z}$-valued function $\tilde{h_{1}}$ as in the lemma. Then, anytime there is an edge $u v$ satisfying $\left|\tilde{h_{1}}(v)-\tilde{h_{1}}(u)\right|=2$, put a quadri-tile made of the two right triangles adjacent to this edge. This defines a 2-tiling of $R$.

\subsubsection{Second height function}

Consider a triangular quadri-tiling $T$. Let $L(T)$ be its underlying lozenge-withdiagonals tiling, and $h_{1}$ be the first height function on vertices of $T$. The lozenge tiling $\mathrm{L}(T)$ corresponding to $L(T)$ is a 2-tiling of the equilateral triangular lattice $\mathbb{T}$. Moreover $\mathbb{T}$ has a bipartite coloring of its faces, say black and white. Orient the edges around the black faces cclw, edges around the white faces are then oriented cw. Thurston [18] defines the second height function $h_{2}$ as follows: choose a vertex $v_{2}$ of $L(T)$, and set $h_{2}\left(v_{2}\right)=0$. For every other vertex $v$ of $L(T)$, take an edge-path $\gamma_{2}$ from $v_{2}$ to $v$ which follows the boundaries of the lozenges of $L(T)$. The second height function $h_{2}$ changes by \pm 1 along each edge of $\gamma_{2}$ : if an edge is oriented in the direction of the path, then $h_{2}$ increases by 1 , if it is oriented in the opposite direction, then $h_{2}$ decreases by 1 . The value $h_{2}(v)$ is independent of the path $\gamma_{2}$. For convenience, we choose $v_{2}$ to be the same vertex as $v_{1}$, and denote this common vertex by $v_{0}$, so that $h_{1}\left(v_{0}\right)=h_{2}\left(v_{0}\right)=0$. An analog to Lemma 3 gives a bijection between second height functions and lozenge tilings of the plane, hence we deduce that triangular quadri-tilings are characterized by $h_{1}$ and $h_{2}$.

Let us define a natural value for the second height function at the vertex in the center of the lozenges of $\mathrm{L}(T)$. When going cclw around the vertices of a lozenge $\ell$ of $\mathrm{L}(T)$, starting from the smallest value of $h_{2}$ say $h$, vertices take on successive values $h, h+1, h+2, h+1$, so that we assign value $h+1$ to the vertex in the center of the lozenge $\ell$. An example of computation of $h_{2}$ is given in Fig. 4.

In Thurston's geometric interpretation [18], a lozenge tiling is seen as a surface $S$ in $\mathbb{Z}^{3}$ (where the diagonals of the cubes are orthogonal to the plane) that has been projected orthogonally to the plane. The surface $S$ is determined by the height function $h_{2}$. In a similar way, a triangular quadri-tiling of the plane $T$ can be seen as a surface $S_{1}$ in a 4-dimensional space that has been projected to the plane; $S_{1}$ can also be projected to $\widetilde{\mathbb{Z}}^{3}$ ( $\widetilde{\mathbb{Z}}^{3}$ is the space $\mathbb{Z}^{3}$ where cubes are drawn with diagonals on their faces), and one obtains a surface $S_{2}$. When projected to the plane, $S_{2}$ is the underlying lozenge-with-diagonals tiling $L(T)$. 


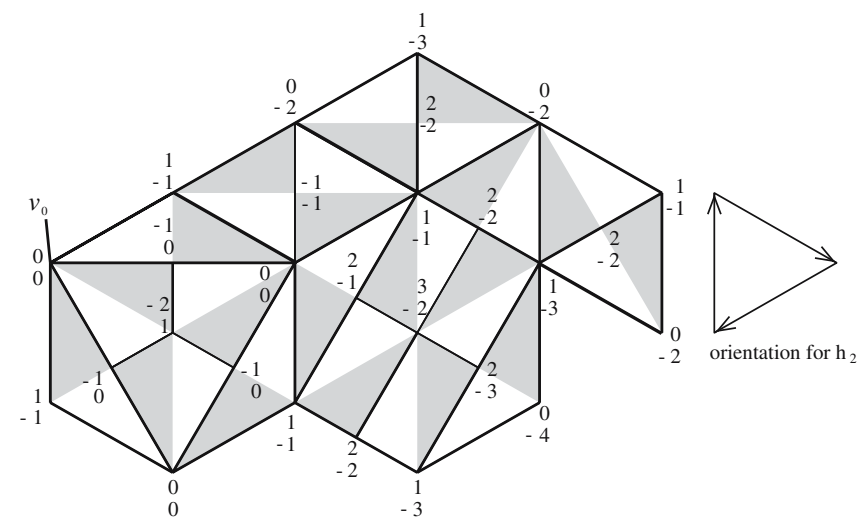

Fig. 4. Triangular quadri-tiling with height functions $h_{1}$ (above) and $h_{2}$ (below)

\subsection{Elementary operations}

Consider a finite simply connected sub-graph $G$ of the equilateral triangular lattice $\mathbb{T}$, and let $\partial G$ be the cycle of $G$ consisting of its boundary edges. Denote by $\mathcal{Q}(\partial G)$ the set of triangular quadri-tilings whose underlying tilings are lozenge tilings of $G$. Let $\mathrm{L}^{1}$ be a lozenge tiling of $G$, and $L^{1}$ be the corresponding lozenge-with-diagonals tiling. Then using the bijection between the first height function and 2-tilings of $L^{1}$ we obtain, in exactly the same way as Elkies et al. [3] have for domino tilings, the following lemma:

Lemma 4. Every 2-tiling of $L^{1}$ can be transformed into any other by a finite sequence of the following operations, (Fig. 5, in brackets is the number of possible orientations for the graph corresponding to the operation):
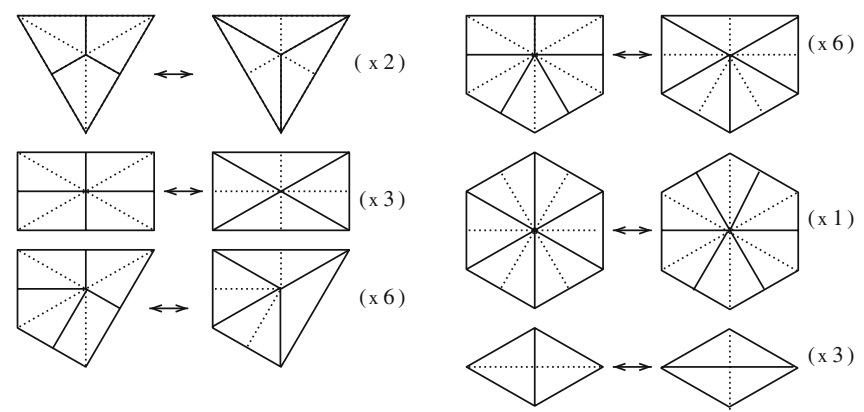

Fig. 5. Quadri-tile operations

Let us call quadri-tile operations the 21 operations described in Lemma 4. Moreover, every lozenge tiling of $G$ can be transformed into any other by a finite sequence of lozenge operations (Fig. 6):

Note that if $L$ is any lozenge tiling of $G$, then $L$ is quadri-tilable with quadritiles obtained by cutting in two every lozenge along one of its diagonals. Moreover, 


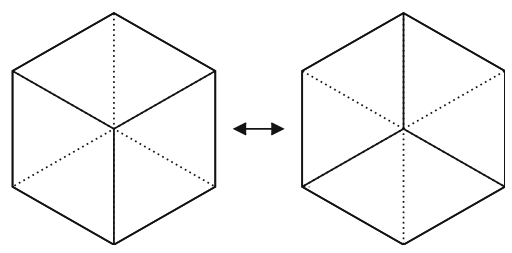

Fig. 6. Lozenge operations

when one performs a lozenge operation on such a quadri-tiling, one still obtains a quadri-tiling of $\mathcal{Q}(\partial G)$. Let us call elementary operations the quadri-tile operations and the lozenge operations performed on quadri-tilings as described above. Then we have:

Lemma 5. Every quadri-tiling of $\mathcal{Q}(\partial G)$ can be transformed into any other by a finite sequence of elementary operations.

Proof. This results from Lemma 4, and the above observation.

\section{Gibbs measure on quadri-tilings}

This section aims at giving a precise statement of Theorem 1 of Sect. 1 (see Theorem 4 below). Sections 3.1 and 3.2 are taken from [8] and give a precise definition of an isoradial graph, the critical weight function, the Dirac and inverse Dirac operator. Section 3.3 consists in the statement of Theorem 4.

\subsection{Isoradial graphs and critical weight function}

The definition of the critical weight function follows [8]. It is defined on edges of graphs satisfying a geometric condition called isoradiality: all faces of an isoradial graph are inscribable in a circle, and all circumcircles have the same radius.

Note that if $\mathrm{R}$ is a rhombus tiling of the plane, then the corresponding rhombus-with-diagonals tiling $R$ is an isoradial graph. Let us consider the embedding of the dual graph $R^{*}$ (the same notation is used for the one-skeleton of a graph and its embedding) where the dual vertices are the circumcenters of the corresponding faces. Then $R^{*}$ is also an isoradial graph and the circumcenters of the faces are the vertices of $R$.

To each edge $e$ of $R^{*}$, we associate a unit side-length rhombus $R(e)$ whose vertices are the vertices of $e$ and the vertices of its dual edge. Let $\widetilde{R}=\cup_{e \in R^{*}} R(e)$. Note that the dual edges corresponding to the boundary edges of the rhombi of $R$ have length zero, and that the rhombi associated to these edges are degenerated.

For each edge $e$ of $R^{*}$, define $v(e)=2 \sin \theta$, where $2 \theta$ is the angle of the rhombus $R(e)$ at the vertex it has in common with $e ; \theta$ is called the rhombus angle of the edge $e$. Note that $v(e)$ is the length of $e^{*}$, the dual edge of $e$. The function $v$ is called the critical weight function. 


\subsection{Dirac and inverse Dirac operator}

Results and definitions of this section are due to Kenyon [8], see also Mercat [14]. Note that this section (as the previous one) is true for general isoradial graphs with bipartite dual graphs.

Let $\mathrm{R}$ be a rhombus tiling of the plane, then by Lemma $2, R^{*}$ is a bipartite graph. Let $B$ (resp. $W$ ) be the set of black (resp. white) vertices of $R^{*}$, and denote by $v$ the critical weight function on the edges of $R^{*}$. The Hermitian matrix $K$ indexed by the vertices of $R^{*}$ is defined as follows. If $v_{1}$ and $v_{2}$ are not adjacent $K\left(v_{1}, v_{2}\right)=0$. If $w \in W$ and $b \in B$ are adjacent vertices, then $K(w, b)=\overline{K(b, w)}$ is the complex number of modulus $v(w b)$ and direction pointing from $w$ to $b$. If $w$ and $b$ have the same image in the plane, then $|K(w, b)|=2$, and the direction of $K(w, b)$ is that which is perpendicular to the corresponding dual edge, and has sign determined by the local orientation. The infinite matrix $K$ defines the Dirac operator $K: \mathbb{C}^{V\left(R^{*}\right)} \rightarrow \mathbb{C}^{V\left(R^{*}\right)}$, by

$$
(K f)(v)=\sum_{u \in R^{*}} K(v, u) f(u),
$$

where $V\left(R^{*}\right)$ denotes the set of vertices of the graph $R^{*}$.

The inverse Dirac operator $K^{-1}$ is defined to be the operator satisfying:

1. $K K^{-1}=\mathrm{Id}$.

2. $K^{-1}(b, w) \rightarrow 0$, when $|b-w| \rightarrow \infty$.

Kenyon [8] obtains an explicit expression for $K^{-1}$. Before stating his theorem, we need to define the rational functions $f_{w v}(z)$. Let $w$ be a white vertex of $R^{*}$. For every other vertex $v$, define $f_{w v}(z)$ as follows. Let $w=v_{0}, v_{1}, v_{2}, \ldots, v_{k}=v$ be an edge-path of $\widetilde{R}$, from $w$ to $v$. Each edge $v_{j} v_{j+1}$ has exactly one vertex of $R^{*}$ (the other is a vertex of $R$ ). Direct the edge away from this vertex if it is white, and towards this vertex if it is black. Let $\mathrm{e}^{i \alpha_{j}}$ be the corresponding vector in $\widetilde{R}$ (which may point contrary to the direction of the path), then $f_{w v}$ is defined inductively along the path, starting from

$$
f_{w w}(z)=1 .
$$

If the edge leads away from a white vertex, or towards a black vertex, then

$$
f_{w v_{j+1}}(z)=\frac{f_{w v_{j}}(z)}{z-\mathrm{e}^{i \alpha_{j}}},
$$

else, if it leads towards a white vertex, or away from a black vertex, then

$$
f_{w v_{j+1}}(z)=f_{w v_{j}}(z)\left(z-\mathrm{e}^{i \alpha_{j}}\right)
$$

The function $f_{w v}(z)$ is well defined (i.e. independent of the edge-path of $\widetilde{R}$ from $w$ to $v$ ), because the multipliers for a path around a rhombus of $\widetilde{R}$ come out to 1 . For a black vertex $b$ the value $K^{-1}(b, w)$ will be the sum over the poles of $f_{w b}(z)$ of the residue of $f_{w b}$ times the angle of $z$ at the pole. However, there is an ambiguity in the choice of angle, which is only defined up to a multiple of $2 \pi$. To make 
this definition precise, angles are assigned to the poles of $f_{w b}(z)$. Working on the branched cover of the plane, branched over $w$, so that for each black vertex $b$ in this cover, a real angle $\theta_{0}$ is assigned to the complex vector $b-w$, which increases by $2 \pi$ when $b$ winds once around $w$. In the branched cover of the plane, a real angle in $\left[\theta_{0}-\pi+\Delta, \theta_{0}+\pi-\Delta\right]$ can be assigned to each pole of $f_{w b}$, for some small $\Delta>0$.

Theorem 3. [8] There exists a unique $K^{-1}$ satisfying the above two properties, and $K^{-1}$ is given by:

$$
K^{-1}(b, w)=\frac{1}{4 \pi^{2} i} \int_{C} f_{w b}(z) \log z \mathrm{~d} z
$$

where $C$ is a closed contour surrounding cclw the part of the circle $\left\{\mathrm{e}^{i \theta} \mid \theta \in\left[\theta_{0}-\pi\right.\right.$ $\left.\left.+\Delta, \theta_{0}+\pi-\Delta\right]\right\}$, which contains all the poles of $f_{w b}$, and with the origin in its exterior, see Fig. 7.

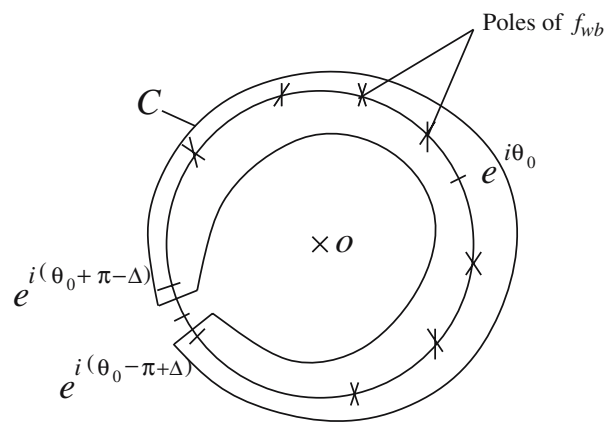

Fig. 7. An example of contour $C$

The remarkable property of $K^{-1}(b, w)$ is that it only depends on the local geometry of the graph, i.e. on an edge-path from $w$ to $b$.

\subsection{Statement of result}

Let $\mathrm{R}$ be a rhombus tiling of the plane, and $R$ be the corresponding rhombus-withdiagonals tiling. Suppose that the critical weight function $v$ is assigned to edges of $R^{*}$, and let $K$ be the Dirac operator indexed by the vertices of $R^{*}$. Moreover, recall that if $e_{1}=w_{1} b_{1}, \ldots, e_{k}=w_{k} b_{k}$ is a subset of edges of $R^{*}$, then the cylinder $\left\{e_{1}, \ldots, e_{k}\right\}$ is defined to be the set of dimer configurations of $R^{*}$ which contain these edges. Let $\mathcal{A}$ be the field consisting of the empty set and of the finite disjoint unions of cylinders. Denote by $\sigma(\mathcal{A})$ the $\sigma$-field generated by $\mathcal{A}$. 
Theorem 4. There is a probability measure $\mu^{R}$ on $\left(\mathcal{M}\left(R^{*}\right), \sigma(\mathcal{A})\right)$ such that for every cylinder $\left\{e_{1}, \ldots, e_{k}\right\}$ of $R^{*}$,

$$
\mu^{R}\left(e_{1}, \ldots, e_{k}\right)=\left(\prod_{i=1}^{k} K\left(w_{i}, b_{i}\right)\right) \underset{1 \leq i, j \leq k}{\operatorname{det}_{1}}\left(K^{-1}\left(b_{i}, w_{j}\right)\right) .
$$

Moreover $\mu^{R}$ is a Gibbs measure on $\mathcal{M}\left(R^{*}\right)$. When $R^{*}$ is doubly periodic, $\mu^{R}$ is the unique Gibbs measure which has minimal free energy per fundamental domain among the two-parameter family of ergodic Gibbs measures of [9].

From the proof, it appears that Theorem 4 is in fact true for all doubly periodic isoradial graphs with bipartite dual graphs. We refer to Sect. 1 for comments on Theorem 4.

\section{Proof of Theorem 4}

The proof of Theorem 4 uses Propositions 1 and 2 below. Proposition 1 is a geometric property of rhombus tilings and is the subject of Sect. 4.1. Proposition 2 concerns the convergence of the Boltzmann measure on some appropriate toroidal graphs, it is the subject of Sect. 4.3. In Sect. 4.2, we introduce the real Dirac operator and its inverse. This operator is related to the Dirac operator and is needed for the proof of Proposition 2. Theorem 4 is then proved in Sect. 4.4.

\subsection{Geometric property of rhombus tilings}

Proposition 1. Let $\mathrm{R}$ be a rhombus tiling of the plane, then any finite simply connected sub-graph $\mathrm{P}$ of $\mathrm{R}$ can be embedded in a periodic rhombus tiling $\mathrm{S}$ of the plane.

Proof. This proposition is a direct consequence of Lemmas 6, 7 and Theorem 5 below.

The notion of train-track has been introduced by Mercat [13], see also Kenyon and Schlenker $[8,11]$. A train-track of a rhombus tiling is a path of rhombi (each rhombus being adjacent along an edge to the previous rhombus) which does not turn: on entering a rhombus, it exits across the opposite edge. Train-tracks are assumed to be maximal in the sense that they extend in both directions as far as possible. Thus train-tracks of rhombus tilings of the plane are bi-infinite. Each rhombus in a train-track has an edge parallel to a fixed unit vector $e$, called the transversal direction of the train-track. Let us denote by $t_{e}$ the train-track of transversal direction $e$. In an oriented train-track (i.e. the edges of the two parallel boundary paths of the train-track have the same given orientation), we choose the direction of $e$ so that when the train-track runs in the direction given by the orientation, $e$ points from the right to the left. The vector $e$ is called the oriented transversal direction of the oriented train-track. A train-track cannot cross itself, and two different train-tracks can cross at most once. A finite simply connected sub-graph $P$ of a rhombus tiling of the plane $\mathrm{R}$ is train-track-convex, if every train-track of $\mathrm{R}$ that intersects $\mathrm{P}$ crosses the boundary of $\mathrm{P}$ twice exactly. 
Lemma 6. Let $\mathrm{R}$ be a rhombus tiling of the plane, then any finite simply connected sub-graph $\mathrm{P}$ of $\mathrm{R}$ can be completed by a finite number of rhombi of $\mathrm{R}$ in order to become train-track-convex.

Proof. Let $e_{1}, \ldots, e_{m}$ be the boundary edges of $\mathrm{P}$. Every rhombus of $\mathrm{P}$ belongs to two train-tracks of $R$, each of which can be continued in both directions up to the boundary of $P$. In both directions the intersection of each of the train-tracks and the boundary of $P$ is an edge parallel to the transversal direction of the train-track. Thus, to take into account all train-tracks of $R$ that intersect $P$, it suffices to consider for every $i$ the train-tracks $t_{e_{i}}$ associated to the boundary edges of P. Consider the following algorithm (see Fig. 8).

Set $Q_{1}=P$.

For $i=1, \ldots, m$, do the following:

Consider the train-track $t_{e_{i}}$, and let $2 n_{i}$ be the number of times $t_{e_{i}}$ intersects the boundary of $\mathrm{Q}_{i}$.

- If $n_{i}>1$ : there are $n_{i}-1$ portions of $t_{e_{i}}$ that are outside of $\mathrm{Q}_{i}$, denote them by $t_{e_{i}}{ }^{1}, \ldots, t_{e_{i}}{ }^{n_{i}-1}$. Then, since $Q_{i}$ is simply connected, for every $j, \mathrm{R} \backslash\left(\mathrm{Q}_{i} \cup t_{e_{i}}{ }^{j}\right)$ is made of two disjoint sub-graphs of $\mathrm{R}$, one of which is finite (it might be empty in the case where one of the two parallel boundary paths of $t_{e_{i}}{ }^{j}$ is part of the boundary path of $\mathrm{Q}_{i}$ ). Denote by $g_{e_{i}}{ }^{j}$ the simply connected sub-graph of $\mathrm{R}$ made of the finite sub-graph of $\mathrm{R} \backslash\left(\mathrm{Q}_{i} \cup t_{e_{i}}{ }^{j}\right)$ and of $t_{e_{i}}{ }^{j}$. Denote by $b_{e_{i}}{ }^{j}$ the portion of the boundary of $\mathrm{Q}_{i}$ which bounds $g_{e_{i}}{ }^{j}$. Replace $\mathrm{Q}_{i}$ by $\mathrm{Q}_{i+1}=\mathrm{Q}_{i} \cup\left(\cup_{j=1}^{n_{i}-1} g_{e_{i}}{ }^{j}\right)$. By this construction $t_{e_{i}}$ intersects the boundary of $Q_{i+1}$ exactly twice, and $Q_{i+1}$ is simply connected.

- If $n_{i}=1$ : set $Q_{i+1}=Q_{i}$.

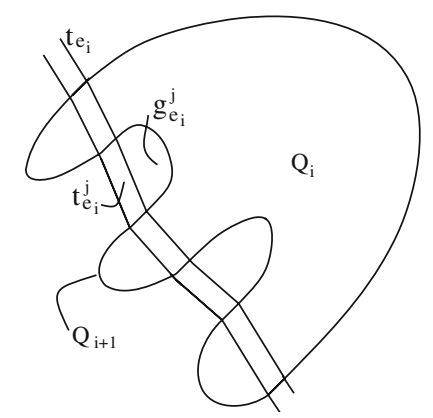

Fig. 8. One step of the algorithm

Let us show that at every step the train-tracks of $R$ that intersect $Q_{i}$ and $Q_{i+1}$ are the same. By construction, boundary edges of $Q_{i+1}$ are boundary edges of $Q_{i}$ and of $t_{e_{i}}{ }^{j}$, for every $j$. Let $f$ be an edge on the boundary of $Q_{i+1}$, but not of $\mathrm{Q}_{i}$, that is $f$ is on the boundary of $t_{e_{i}}{ }^{j}$ for some $j$, thus $t_{f}$ crosses $g_{e_{i}}{ }^{j}$. Since two train-tracks cross at most once, $t_{f}$ has to intersect $b_{e_{i}}{ }^{j}$, which means $t_{f}$ also crosses $Q_{i}$. From this we also conclude that if a train-track intersects the boundary of $Q_{i}$ twice, then it also intersects the boundary of $Q_{i+1}$ twice. 
Thus all train-tracks that intersect $Q_{m+1}$ cross its boundary exactly twice, and $\mathrm{Q}_{m+1}$ contains $\mathrm{P}$.

Lemma 7. Let $\mathrm{R}$ be a rhombus tiling of the plane. Then any finite simply connected train-track-convex sub-graph $\mathrm{P}$ of $\mathrm{R}$ can be completed by a finite number of rhombi in order to become a convex polygon $\mathrm{Q}$, whose opposite boundary edges are parallel.

Proof. Let $e_{1}, \ldots, e_{m}$ be the boundary edges of $\mathrm{P}$ oriented cclw. Since $\mathrm{P}$ is traintrack-convex, the train-tracks $t_{e_{1}}, \ldots, t_{e_{m}}$ intersect the boundary of $\mathrm{P}$ twice, so that there are pairs of parallel boundary edges. Let us assume that the transversal directions of the train-tracks are all distinct (if this is not the case, one can always perturb the graph a little so that it happens). Let us also denote by $t_{e_{1}}, \ldots, t_{e_{m}}$ the portions of the bi-infinite train-tracks of $R$ in $P$. In what follows, indices will be denoted cyclically, that is $e_{j}=e_{m+j}$. Write $x_{j}$ (resp. $y_{j}$ ) for the initial (resp. end) vertex of an edge $e_{j}$.

Let $e_{i}, e_{i+1}$ be two adjacent boundary edges of P. Consider the translate $e_{i+1}^{t}$ of $e_{i+1}$ so that the initial vertex of $e_{i+1}^{t}$ is adjacent to the initial vertex of $e_{i}$. Then we define the turning angle from $e_{i}$ to $e_{i+1}$ (also called exterior angle) to be the angle $\widehat{e_{i} e_{i+1}^{t}}$, and we denote it by $\theta_{e_{i}, e_{i+1}}$. If $e_{i}, e_{j}$ are two boundary edges, then the turning angle from $e_{i}$ to $e_{j}$ is defined by $\sum_{\alpha=i}^{j-1} \theta_{e_{\alpha}, e_{\alpha+1}}$, and is denoted by $\theta_{e_{i}, e_{j}}$.

\section{Properties}

1. $\sum_{\alpha=1}^{m} \theta_{e_{\alpha}, e_{\alpha+1}}=2 \pi$.

2. If $e_{i}, e_{j}$ are two boundary edges, and if $\gamma=\left\{f_{1}, \ldots, f_{n}\right\}$ is an oriented edgepath in $\mathrm{P}$ from $y_{i}$ to $x_{j}$, then $\theta_{e_{i}, e_{j}}=\theta_{e_{i}, f_{1}}+\sum_{\alpha=1}^{n-1} \theta_{f_{\alpha}, f_{\alpha+1}}+\theta_{f_{n}, e_{j}}$.

3. If $e_{i}$ is a boundary edge of $\mathrm{P}$, and $e_{k}$ is the second boundary edge at which $t_{e_{i}}$ intersects the boundary of $\mathrm{P}$, then $\theta_{e_{i}, e_{k}}=\pi$. Thus $e_{k}$ and $e_{i}$ are oriented in the opposite direction, and we denote $e_{k}$ by $e_{i}{ }^{-1}$.

4. $P$ is convex, if and only if every train-track of $P$ crosses every other train-track of $P$.

We first end the proof of Lemma 7, and then prove Properties 1 to 4.

Note that Properties 1 and 2 are true for any finite simply connected sub-graph of R.

The number of train-tracks intersecting $\mathrm{P}$ is $n=m / 2$. So that if every traintrack crosses every other train-track, the total number of crossings is $n(n-1) / 2$. Consider the following algorithm (see Fig. 9 for an example).

Set $Q_{1}=P, n_{1}=$ the number of train-tracks that cross in $Q_{1}$.

For $i=1,2, \ldots$ do the following:

- If $n_{i}=n(n-1) / 2$ : then by Property $4, \mathrm{Q}_{i}$ is convex.

- If $n_{i}<n(n-1) / 2$ : then by Property $4, \theta_{e_{j_{i}}, e_{j_{i}+1}}<0$ for some $j_{i} \in$ $\{1, \ldots, m\}$. Add the rhombus $\ell_{j_{i}}$ of parallel directions $e_{j_{i}}, e_{j_{i}+1}$ along the boundary of $Q_{i}$. Set $Q_{i+1}=Q_{i} \cup \ell_{j i}$, and rename the boundary edges $e_{1}, \ldots, e_{m}$ in cclw order. Then the number of train-tracks that cross in $\mathrm{Q}_{i+1}$ is $n_{i}+1$, set $n_{i+1}=n_{i}+1$. Note that if Property 4 is true for $\mathrm{Q}_{i}$, it stays true for $Q_{i+1}$, and note that the same train-tracks intersect $Q_{i}$ and $Q_{i+1}$. 


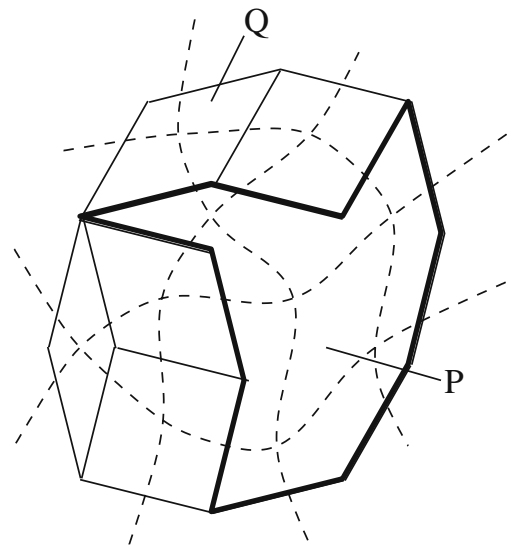

Fig. 9. Example of application of the algorithm

For the algorithm to be able to add the rhombus $\ell_{j_{i}}$ at every step, we need to check that:

$$
\forall i, \theta_{e_{j_{i}-1}, e_{j_{i}+1}}>-\pi \text {, and } \theta_{e_{j_{i}}, e_{j_{i}+2}}>-\pi .
$$

Assume we have proved that for any finite simply connected train-track-convex sub-graph $\mathrm{P}$ of $\mathrm{R}$ we have:

$$
\forall i, j, \theta_{e_{i}, e_{j}}>-\pi .
$$

Then Properties 1 and 2 imply that if (4) is true for $Q_{i}$ it stays true for $Q_{i+1}$, moreover (4) implies (3). So let us prove (4) by induction on the number of rhombi contained in $\mathrm{P}$. If $\mathrm{P}$ is a rhombus, then (4) is clear. Now assume $\mathrm{P}$ is made of $k$ rhombi. Consider the train-tracks in $\mathrm{P}$ adjacent to the boundary (every boundary edge $e$ of $\mathrm{P}$ belongs to a rhombus of $\mathrm{P}$ which has parallel directions $e$ and $f$; for every boundary edge $e$, the train-track of transversal direction $f$ is the traintrack adjacent to the boundary). Denote the train-tracks adjacent to the boundary by $t_{1}, \ldots, t_{p}$ in cclw order, and write $f_{\beta}$ for the oriented transversal direction of $t_{\beta}$ (when the boundary edge-path of $\mathrm{P}$ is oriented cclw). Consider two adjacent boundary edges $e_{i}, e_{i+1}$ of $\mathrm{P}$ that do not belong to the same boundary train-track. That is $e_{i}$ belongs to $t_{\beta}$, and $e_{i+1}$ to $t_{\beta+1}$. Then either $\widehat{f_{\beta} f_{\beta+1}}<0$ or $\widehat{f_{\beta} f_{\beta+1}}>0$, in the second case $t_{\beta}$ and $t_{\beta+1}$ cross and their intersection is a rhombus $\ell_{\beta}$ of $\mathrm{P}$. The rhombus $\ell_{\beta}$ has boundary edges $e_{i}, e_{i+1}$, and $f_{\beta+1}=e_{i}^{-1}, f_{\beta}^{-1}=e_{i+1}{ }^{-1}$. Now Property 1 implies that $\sum_{\beta=1}^{p-1} \widehat{f_{\beta} f_{\beta+1}}=2 \pi$, so that there always exists $\beta_{0}$ such that $\widehat{f_{\beta_{0}} f_{\beta_{0}+1}}>0$. Removing $\ell_{\beta_{0}}$ from $\mathrm{P}$ and using the assumption that $\mathrm{P}$ is train-track-convex, we obtain a graph $\mathrm{P}^{\prime}$ made of $k-1$ rhombi which is traintrack-convex. By induction, $\theta_{e, f}>-\pi$ for every boundary edge of $\mathrm{P}^{\prime}$, and using Property 2, we conclude that this stays true for $\mathrm{P}$.

Denote by $\mathrm{Q}$ the convex polygon obtained from $\mathrm{P}$ by the algorithm, and assume that opposite boundary edges are not parallel. Then there are indices $i$ and $j$ such that $e_{i}$ comes before $e_{j}$, and $e_{j}^{-1}$ comes before $e_{i}^{-1}$. This implies that $\theta_{e_{i}, e_{j}}=$ $-\theta_{e_{j}-1, e_{i}-1}$, so that one of the two angles is negative, which means $\mathrm{Q}$ cannot be 
convex. Thus we have a contradiction, and we conclude that opposite boundary edges of $Q$ are parallel.

\section{Proof of Properties 1 to 4.}

1. and 2. are straightforward.

3. When computing $\theta_{e_{i}, e_{k}}$ along the boundary edge-path of $t_{e_{i}}$ we obtain $\pi$, so by Property 2 we deduce that $\theta_{e_{i}, e_{k}}=\pi$ in P.

4. $\mathrm{P}$ is convex if and only if, for every $i, \theta_{e_{i}, e_{i+1}}>0$, which is equivalent to saying that, for every $i \neq j, \theta_{e_{i}, e_{j}}>0$. Therefore Property 4 is equivalent to proving that $\theta_{e_{i}, e_{j}}>0$, for every $i \neq j$, if and only if every train-track of $\mathrm{P}$ crosses every other train-track of $P$.

Assume there are two distinct train-tracks $t_{e_{\ell}}$ and $t_{e_{k}}$ that do not cross in P. Then, in cclw order around the boundary of $\mathrm{P}$, we have either $e_{\ell}, e_{k}{ }^{-1}, e_{k}, e_{\ell}{ }^{-1}$ or $e_{\ell}, e_{k}, e_{k}{ }^{-1}, e_{\ell}{ }^{-1}$. It suffices to solve the second case, the first case being similar. By Property $1, \theta_{e_{\ell}, e_{k}}+\theta_{e_{k}, e_{k}-1}+\theta_{e_{k}-1, e_{\ell}-1}+\theta_{e_{\ell}-1, e_{\ell}}=2 \pi$. Moreover by Property 3, $\theta_{e_{k}, e_{k}-1}=\theta_{e_{\ell}-1, e_{\ell}}=\pi$, which implies $\theta_{e_{\ell}, e_{k}}=-\theta_{e_{k}{ }^{-1}, e_{\ell}-1}$. Since all train-tracks have different transversal directions, either $\theta_{e_{\ell}, e_{k}}$ or $\theta_{e_{k}-1, e_{\ell}-1}$ is negative.

Now take two boundary edges $e_{i}, e_{j}$ of $\mathrm{P}$ (with $i \neq j$, and $e_{j} \neq e_{i}{ }^{-1}$ ), and assume the train-tracks $t_{e_{i}}, t_{e_{j}}$ cross inside $\mathrm{P}$. Then in cclw order around the boundary of $\mathrm{P}$, we have either $e_{i}, e_{j}^{-1}, e_{i}^{-1}, e_{j}$, or $e_{i}, e_{j}, e_{i}^{-1}, e_{j}^{-1}$. It suffices to solve the second case since the first case can be deduced from the second one. The intersection of $t_{e_{i}}$ and $t_{e_{j}}$ is a rhombus $\ell$. Let $\tilde{e}_{j}^{-1}$ (resp. $\tilde{e}_{i}^{-1}$ ) be the boundary edge of $\ell$ parallel and closest to $e_{j}$ (resp. $e_{i}$ ), oriented in the opposite direction, then $\theta_{\tilde{e}_{j}^{-1}, \tilde{e}_{i}^{-1}}<0$. Let $\gamma_{j}$ (resp. $\gamma_{i}$ ) be the boundary edge-path of $t_{e_{j}}$ (resp. $t_{e_{i}}$ ) from $y_{j}$ to $\tilde{x}_{j}$ (resp. from $\tilde{y}_{i}$ to $x_{i}$ ), and let $\mathrm{Q}$ be the sub-graph of $\mathrm{R}$ whose boundary is $e_{i}, e_{i+1}, \ldots, e_{j}, \gamma_{j}, \tilde{e}_{j}^{-1}, \tilde{e}_{i}^{-1}, \gamma_{i}$. Since $t_{e_{i}}$ and $t_{e_{j}}$ intersect the boundary of $\mathrm{P}$ twice, they also intersect the boundary of $\mathrm{Q}$ twice. Moreover $t_{e_{i}}$ and $t_{e_{j}}$ do not cross in $\mathrm{Q}$, so that $\theta_{e_{i}, e_{j}}=-\theta_{\tilde{e}_{j}^{-1}, \tilde{e}_{i}^{-1}}>0$.

Theorem 5. Any convex $2 n$-gon $\mathrm{Q}$ whose opposite boundary edges are parallel and of the same length can be embedded in a periodic tiling of the plane by $\mathrm{Q}$ and rhombi.

Proof. Let $e_{1}, \ldots, e_{n}, e_{1}^{-1}, \ldots, e_{n}^{-1}$ be the boundary edges of the polygon $\mathrm{Q}$ oriented cclw. If $n \leq 3$, then $\mathrm{Q}$ is either a rhombus or a hexagon, and it is straightforward that the plane can be tiled periodically with $Q$.

If $n>4$, for $k=1, \ldots, n-3$, do the following (see Fig. 10): along $e_{n-k}$ add the finite train-track $\tilde{t}_{e_{n-k}}$ of transversal direction $e_{n-k}$, going away from $\mathrm{Q}$, whose boundary edges starting from the boundary of $Q$ are:

$$
e_{1}, \underbrace{e_{2}, e_{1}}, \underbrace{e_{3}, e_{2}, e_{1}}, \ldots, \underbrace{e_{n-k-2}, \ldots, e_{1}} .
$$

Since the polygon $\mathrm{Q}$ is convex, the rhombi that are added are well defined, moreover the intersection of $\tilde{t}_{e_{i}}$ and the boundary of $\mathrm{Q}$ is the edge $e_{i}$, and $\tilde{t}_{e_{i}}$ does 


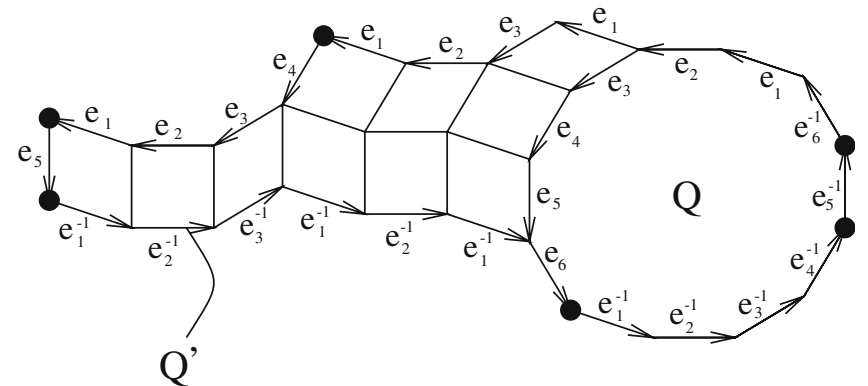

Fig. 10. Fundamental domain of a periodic tiling of the plane by dodecagons and rhombi

not cross $\tilde{t}_{e_{j}}$ when $i \neq j$. So we obtain a new polygon $\mathrm{Q}^{\prime}$ made of $\mathrm{Q}$ and rhombi, whose boundary edge-path is $\gamma_{1}, \ldots, \gamma_{6}$ (when starting from the edge $e_{n}{ }^{-1}$ of Q), where:

$$
\begin{aligned}
& \gamma_{1}=e_{n}^{-1}, e_{1}, \underbrace{e_{2}, e_{1}}, \underbrace{e_{3}, e_{2}, e_{1}}, \ldots, \underbrace{e_{n-3}, \ldots, e_{1}}, \\
& \gamma_{2}=e_{n-2}, \ldots, e_{1}, \\
& \gamma_{3}=e_{n-1}, \\
& \gamma_{4}=\underbrace{e_{1}^{-1}, \ldots, e_{n-3}{ }^{-1}}, \ldots, \underbrace{e_{1}^{-1}, e_{2}^{-1}}, e_{1}^{-1}, e_{n}, \\
& \gamma_{5}=e_{1}^{-1}, \ldots, e_{n-2}^{-1}, \\
& \gamma_{6}=e_{n-1}^{-1} .
\end{aligned}
$$

Noting that $\gamma_{4}=\gamma_{1}^{-1}, \gamma_{5}=\gamma_{2}^{-1}, \gamma_{6}=\gamma_{3}^{-1}$, and using the fact that the plane can be tiled periodically with hexagons which have parallel opposite boundary edges, we deduce that the plane can be tiled with $\mathrm{Q}^{\prime}$, that is it can be tiled periodically by $\mathrm{Q}$ and rhombi.

Remark 1. It was pointed out by the referee that Theorem 5 might be known already. After a second look at the extensive literature on tilings, we were not able to find a reference, but any information is of course welcome.

\subsection{Real Dirac and inverse real Dirac operator}

In the whole of this section we let $\mathrm{R}$ be a rhombus tiling of the plane, and $R$ be the corresponding rhombus-with-diagonals tiling. Assume that the critical weight function $v$ is assigned to edges of $R^{*}$, and denote by $K$ the Dirac operator indexed by the vertices of $R^{*}$.

The proof of Theorem 4 requires to take the limit of Boltzmann measures on some appropriate toroidal graphs (see Sect. 4.3). In order to do this, we need to introduce the real Dirac operator denoted by K. Both the Dirac operator $K$ and the real Dirac operator $\mathrm{K}$ are represented by infinite weighted adjacency matrices indexed by the vertices of $R^{*}$. For $K$, the edges of $R^{*}$ are un-oriented and weighted by their critical weight times a complex number of modulus 1 . For K, edges of $R^{*}$ 
are oriented with a clockwise odd orientation, and are weighted by their critical weight. Both weight functions yield the same probability measure on finite simply connected sub-graphs of $R^{*}$, but, and this is the reason why we introduce the real Dirac operator, these weights do not yield the same probability distribution on toroidal sub-graphs of $R^{*}$.

The structure of this section is close to that of Sect. 3.2. We first define the real Dirac operator $\mathrm{K}$ and its inverse $\mathrm{K}^{-1}$ and then, using results of [8], we prove the existence and uniqueness of $\mathrm{K}^{-1}$ by giving an explicit expression for $\mathrm{K}^{-1}$. Note that this section is actually true for general isoradial graphs with bipartite dual graphs.

\subsubsection{Real Dirac operator}

Let us define an orientation of the edges of $R^{*}$. An elementary cycle $C$ of $R^{*}$ is said to be clockwise odd if, when traveling $\mathrm{cw}$ around the edges of $C$, the number of co-oriented edges is odd. Note that since $R^{*}$ is bipartite, the number of contraoriented edges is also odd. Kasteleyn [6] defines the orientation of the graph $R^{*}$ to be clockwise odd if all elementary cycles are clockwise odd. He also proves that, for planar simply connected graphs, such an orientation always exists.

Consider a clockwise odd orientation of the edges of $R^{*}$. Define $\mathrm{K}$ to be the infinite adjacency matrix of the graph $R^{*}$, weighted by the critical weight function $v$. That is, if $v_{1}$ and $v_{2}$ are not adjacent, $\mathrm{K}\left(v_{1}, v_{2}\right)=0$. If $w \in W$ and $b \in B$ are adjacent vertices, then $\mathrm{K}(w, b)=-\mathrm{K}(b, w)=(-1)^{\mathbb{I}_{(w, b)}} v(w b)$, where $\mathbb{I}_{(w, b)}=0$ if the edge $w b$ is oriented from $w$ to $b$, and 1 if it is oriented from $b$ to $w$. The infinite matrix $\mathrm{K}$ defines the real Dirac operator $\mathrm{K}: \mathbb{C}^{V\left(R^{*}\right)} \rightarrow \mathbb{C}^{V\left(R^{*}\right)}$, by

$$
(\mathrm{K} f)(v)=\sum_{u \in R^{*}} \mathrm{~K}(v, u) f(u) .
$$

The matrix $\mathrm{K}$ is also called a Kasteleyn matrix for the underlying dimer model.

\subsubsection{Inverse real Dirac operator}

The inverse real Dirac operator $\mathrm{K}^{-1}$ is defined to be the unique operator satisfying

1. $\mathrm{KK}^{-1}=\mathrm{Id}$.

2. $\mathrm{K}^{-1}(b, w) \rightarrow 0$, when $|b-w| \rightarrow \infty$.

Let us define the rational functions $\mathrm{f}_{w x}(z)$. They are the analogous of the rational functions $f_{w v}(z)$, but are defined for vertices $x \in R^{*}$ (whereas the functions $f_{w v}(z)$ were defined for vertices $\left.v \in \widetilde{R}\right)$. Let $w \in W$, and let $x \in B$ (resp. $x \in W$ ); consider the edge-path $w=w_{1}, b_{1}, \ldots, w_{k}, b_{k}=x$ (resp. $\left.w=w_{1}, b_{1}, \ldots, w_{k}, b_{k}, w_{k+1}=x\right)$ of $R^{*}$ from $w$ to $x$. Let $R\left(w_{j} b_{j}\right)$ be the rhombus associated to the edge $w_{j} b_{j}$, and denote by $w_{j}, x_{j}, b_{j}, y_{j}$ its vertices in cclw order; $\mathrm{e}^{i \alpha_{j}}$ is the complex vector $y_{j}-w_{j}$, and $\mathrm{e}^{i \beta_{j}}$ is the complex vector $x_{j}-w_{j}$. In a similar way, denote by $w_{j+1}, x_{j}^{\prime}, b_{j}, y_{j}^{\prime}$ the vertices of the rhombus 
$R\left(w_{j+1} b_{j}\right)$ in cclw order, then $\mathrm{e}^{i \alpha_{j}^{\prime}}$ is the complex vector $y_{j}^{\prime}-w_{j+1}$, and $\mathrm{e}^{i \beta_{j}^{\prime}}$ is the complex vector $x_{j}^{\prime}-w_{j+1}$. Then $\mathrm{f}_{w x}(z)$ is defined inductively along the path,

$$
\begin{aligned}
\mathrm{f}_{w w}(z) & =1 \\
\mathrm{f}_{w b_{j}}(z) & =\mathrm{f}_{w w_{j}}(z) \frac{(-1)^{\mathbb{I}_{\left(w_{j}, b_{j}\right)}} \mathrm{e}^{i \frac{\alpha_{j}+\beta_{j}}{2}}}{\left(z-\mathrm{e}^{i \alpha_{j}}\right)\left(z-\mathrm{e}^{i \beta_{j}}\right)}, \\
\mathrm{f}_{w w_{j+1}}(z) & =\mathrm{f}_{w b_{j}}(z)(-1)^{\mathbb{I}_{\left(w_{j+1}, b_{j}\right)}} \mathrm{e}^{-i \frac{\alpha_{j}^{\prime}+\beta_{j}^{\prime}}{2}}\left(z-\mathrm{e}^{i \alpha_{j}^{\prime}}\right)\left(z-\mathrm{e}^{i \beta_{j}^{\prime}}\right) .
\end{aligned}
$$

Remark 2. We have the following relations between the real and the complex case.

1. $\forall w \in W, \forall x \in B \cup W, \mathrm{f}_{w x}(z)=\overline{\mathrm{f}_{w x}(0)} f_{w x}(z)$.

2. $\forall w \in W, \forall b \in B$, such that $w$ is adjacent to $b, \mathrm{~K}(w, b)=\mathrm{f}_{w b}(0) K(w, b)$.

Proof. 1. This is a direct consequence of the definitions of the functions $\mathrm{f}_{w x}$ and $f_{w x}$.

2. Let $R(w b)$ be the rhombus associated to the edge $w b$, and let $w, x, b, y$ be its vertices in cclw order. Denote by $\mathrm{e}^{i \alpha}$ the complex vector $y-w$, and by $\mathrm{e}^{i \beta}$ the complex vector $x-w$. Let $\theta$ be the rhombus angle of the edge $w b$. By definition we have,

$$
\begin{aligned}
\mathrm{K}(w, b) & =(-1)^{\mathbb{I}_{(w, b)}} 2 \sin \theta=(-1)^{\mathbb{I}_{(w, b)}} \frac{\mathrm{e}^{i \frac{\alpha-\beta}{2}}-\mathrm{e}^{-i \frac{\alpha-\beta}{2}}}{i}, \\
K(w, b) & =i\left(\mathrm{e}^{i \beta}-\mathrm{e}^{i \alpha}\right), \\
\mathrm{f}_{w b}(0) & =(-1)^{\mathbb{I}_{(w, b)}} \mathrm{e}^{-i \frac{\alpha+\beta}{2}} .
\end{aligned}
$$

Combining the above three equations yields 2 .

Lemma 8. The function $\mathrm{f}_{w x}$ is well defined.

Proof. Showing that the function $\mathrm{f}_{w x}$ is well defined amounts to proving that $\mathrm{f}_{w x}$ is independent of the edge-path of $R^{*}$ from $w$ to $x$. This is equivalent to proving the following: let $w_{1}, b_{1}, \ldots, w_{k}, b_{k}, w_{k+1}=w_{1}$ be the vertices of an elementary cycle $C$ of $R^{*}$, where vertices are enumerated in cclw order; if $\mathrm{f}_{w_{1} w_{1}}(z)=1$ then $f_{w_{1} w_{k+1}}(z)=1$. Let us use the notations introduced in the definition of $f_{w x}$, and denote indices cyclically, that is $k+1 \equiv 1$. By Remark 2, we have

$$
\mathbf{f}_{w_{1} w_{k+1}}(z)=\overline{\mathbf{f}_{w_{1} w_{k+1}}(0)} f_{w_{1} w_{k+1}}(z) \text {. }
$$

Since the function $f_{w x}$ is well defined, $f_{w_{1} w_{k+1}}(z)=1$. Hence, it remains to prove that $\overline{\mathrm{f}_{w_{1} w_{k+1}}(0)}=1$. By definition of $\mathrm{f}_{w x}$, we have

$$
\overline{\mathfrak{f}_{w_{1} w_{k+1}}(0)}=\prod_{j=1}^{k}\left((-1)^{\left(\mathbb{I}_{\left(w_{j}, b_{j}\right)}+\mathbb{I}_{\left(w_{j+1}, b_{j}\right)}\right)} \mathrm{e}^{i \frac{\alpha_{j}+\beta_{j}}{2}} \mathrm{e}^{-i \frac{\alpha_{j}^{\prime}+\beta_{j}^{\prime}}{2}}\right) .
$$


Moreover for every $j, \alpha_{j}^{\prime}=\beta_{j}$, so that

$$
\overline{\mathrm{f}_{w_{1} w_{k+1}}(0)}=\prod_{j=1}^{k}\left((-1)^{\left(\mathbb{I}_{\left(w_{j}, b_{j}\right)}+\mathbb{I}_{\left(w_{j+1}, b_{j}\right)}\right)} \mathrm{e}^{i \frac{\alpha_{j}-\beta_{j}}{2}} \mathrm{e}^{i \frac{\alpha_{j}^{\prime}-\beta_{j}^{\prime}}{2}}\right) .
$$

Let $\theta_{j}$ (resp. $\theta_{j}^{\prime}$ ) be the rhombus angle of the edge $w_{j} b_{j}$ (resp. $w_{j+1} b_{j}$ ), then

$$
\overline{\mathfrak{f}_{w_{1} w_{k+1}}(0)}=(-1)^{\sum_{j=1}^{k}\left(\mathbb{I}_{\left(w_{j}, b_{j}\right)}+\mathbb{I}_{\left(w_{j+1}, b_{j}\right)}\right)} \mathrm{e}^{i \sum_{j=1}^{k}\left(\theta_{j}+\theta_{j}^{\prime}\right)} .
$$

The cycle $C$ corresponds to a face of the graph $R^{*}$. Let $c$ be the circumcenter of this face, and let $\tau_{j}$ (resp. $\left.\tau_{j}^{\prime}\right)$ be the angle of the rhombus $R\left(w_{j} b_{j}\right)\left(\right.$ resp. $\left.R\left(w_{j+1} b_{j}\right)\right)$ at the vertex $c$. Then $\tau_{j}=\pi-2 \theta_{j}$, and $\tau_{j}^{\prime}=\pi-2 \theta_{j}^{\prime}$. Since $\sum_{j=1}^{k}\left(\tau_{j}+\tau_{j}^{\prime}\right)=2 \pi$, we deduce $\sum_{j=1}^{k}\left(\theta_{j}+\theta_{j}^{\prime}\right)=\pi(k-1)$. Hence,

$$
\mathrm{e}^{i \sum_{j=1}^{k}\left(\theta_{j}+\theta_{j}^{\prime}\right)}=-(-1)^{k}
$$

Moreover $\mathbb{I}_{\left(w_{j+1}, b_{j}\right)}=1-\mathbb{I}_{\left(b_{j}, w_{j+1}\right)}$, and $(-1)^{1-\mathbb{I}_{\left(b_{j}, w_{j+1}\right)}}=(-1)^{\mathbb{I}_{\left(b_{j}, w_{j+1}\right)}-1}$, so

$$
(-1)^{\sum_{j=1}^{k}\left(\mathbb{I}_{\left(w_{j}, b_{j}\right)}+\mathbb{I}_{\left(w_{j+1}, b_{j}\right)}\right)}=(-1)^{\sum_{j=1}^{k}\left(\mathbb{I}_{\left(w_{j}, b_{j}\right)}+\mathbb{I}_{\left(b_{j}, w_{j+1}\right)}\right)-k} .
$$

Note that $\sum_{j=1}^{k}\left(\mathbb{I}_{\left(w_{j}, b_{j}\right)}+\mathbb{I}_{\left(b_{j}, w_{j+1}\right)}\right)$ is the number of co-oriented edges encountered when traveling cclw around the cycle $C$. Since the orientation of the edges of $R^{*}$ is clockwise odd, it is also counterclockwise odd, and so this number is odd. This implies

$$
(-1)^{\sum_{j=1}^{k}\left(\mathbb{I}_{\left(w_{j}, b_{j}\right)}+\mathbb{I}_{\left(w_{j+1}, b_{j}\right)}\right)}=-(-1)^{-k} .
$$

The proof is completed by combining Eqs. (5)-(7).

As in the complex case, a real angle in $\left[\theta_{0}-\pi+\Delta, \theta_{0}+\pi-\Delta\right]$ can be assigned to each pole of $\mathrm{f}_{w b}$, for some small $\Delta>0$, where $\theta_{0}$ is the real angle assigned to the vector $b-w$.

Lemma 9. There exists a unique $\mathrm{K}^{-1}$ satisfying the above two properties, and $\mathrm{K}^{-1}$ is given by:

$$
\mathrm{K}^{-1}(b, w)=\frac{1}{4 \pi^{2} i} \int_{C} \mathrm{f}_{w b}(z) \log z \mathrm{~d} z,
$$

where $C$ is a closed contour surrounding cclw the part of the circle $\left\{\mathrm{e}^{i \theta} \mid \theta \in\left[\theta_{0}-\pi\right.\right.$ $\left.\left.+\Delta, \theta_{0}+\pi-\Delta\right]\right\}$, which contains all the poles of $\mathrm{f}_{w b}$, and with the origin in its exterior.

Proof. Let $\mathrm{F}(b, w)$ be the right hand side of (8). Fix a vertex $w_{0} \in W$, and let us prove that $\sum_{b \in B} \mathrm{~K}\left(w_{0}, b\right) \mathrm{F}(b, w)=\delta_{w_{0}}(w)$. Denote by $b_{1}, \ldots, b_{k}$ the black neighbors of $w_{0}$. Using Remark 2 , we obtain for every $j$, 


$$
\begin{gathered}
\mathrm{K}\left(w_{0}, b_{j}\right)=\mathrm{f}_{w_{0} b_{j}}(0) K\left(w_{0}, b_{j}\right), \\
\mathrm{f}_{w b_{j}}(z)=\overline{\mathfrak{f}_{w b_{j}}(0)} f_{w b_{j}}(z) .
\end{gathered}
$$

Moreover $\forall w \in W, \forall b \in B$, we have $\overline{\mathrm{f}_{w b}(0)}=\mathrm{f}_{w b}(0)^{-1}=\mathrm{f}_{b w}(0)$, so that $\mathbf{f}_{w_{0} b_{j}}(0) \overline{\mathbf{f}_{w b_{j}}(0)}=\mathbf{f}_{w_{0} b_{j}}(0) \mathbf{f}_{b_{j} w}(0)=\mathbf{f}_{w_{0} w}(0)$. Hence, using Theorem 3 , we obtain for every $j$,

$$
\mathrm{K}\left(w_{0}, b_{j}\right) \mathrm{F}\left(b_{j}, w\right)=\mathrm{f}_{w_{0} w}(0) K\left(w_{0}, b_{j}\right) K^{-1}\left(b_{j}, w\right) .
$$

Since $\mathrm{K}\left(w_{0}, b\right)=0$ when $w_{0}$ and $b$ are not adjacent, and since $K^{-1}$ is the inverse Dirac operator, we obtain

$$
\begin{aligned}
\sum_{b \in B} \mathrm{~K}\left(w_{0}, b\right) \mathrm{F}(b, w) & =\sum_{j=1}^{k} \mathrm{~K}\left(w_{0}, b_{j}\right) \mathrm{F}\left(b_{j}, w\right), \\
& =\mathrm{f}_{w_{0} w}(0) \sum_{j=1}^{k} K\left(w_{0}, b_{j}\right) K^{-1}\left(b_{j}, w\right) \\
& =\mathrm{f}_{w_{0} w}(0) \delta_{w_{0}}(w)=\delta_{w_{0}}(w) .
\end{aligned}
$$

Uniqueness of $\mathrm{K}^{-1}$ follows from the uniqueness of $K^{-1}$.

\subsection{Convergence of the Boltzmann measure on the torus}

Let $\mathrm{R}$ be a rhombus tiling of the plane, and $R$ be the corresponding rhombus-withdiagonals tiling. Suppose that the critical weight function is assigned to edges of $R^{*}$, and denote by $K$ the Dirac operator indexed by the vertices of $R^{*}$.

Consider a subset of edges $e_{1}=w_{1} b_{1}, \ldots, e_{k}=w_{k} b_{k}$ of $R^{*}$, and let $\mathrm{P}$ be a finite simply connected sub-graph of $\mathrm{R}$ such that $P^{*}$ contains these edges. By Proposition 1, there exists a periodic rhombus tiling of the plane $S$ that contains $P$. Let $S$ be the corresponding rhombus-with-diagonals tiling, and assign the critical weight function to edges of $S^{*}$. Denote by $\Lambda$ the lattice which acts periodically on $S$, and suppose that the dual graph $\bar{S}_{n}^{*}$ of the toroidal graph $\bar{S}_{n}=S / n \Lambda$ is bipartite (this is possible by eventually replacing $\Lambda$ by $2 \Lambda$ ). Denote by $\mu_{n}^{S}$ be the Boltzmann measure on dimer configurations $\mathcal{M}\left(\bar{S}_{n}^{*}\right)$ of $\bar{S}_{n}^{*}$. Then we have,

\section{Proposition 2.}

$$
\lim _{n \rightarrow \infty} \mu_{n}^{S}\left(e_{1}, \ldots, e_{k}\right)=\left(\prod_{i=1}^{k} K\left(w_{i}, b_{i}\right)\right) \underset{1 \leq i, j \leq k}{\operatorname{det}} K^{-1}\left(b_{i}, w_{j}\right) .
$$

Proof. Let us first define an orientation of the edges of $S^{*}$, and the four Kasteleyn matrices $\mathrm{K}_{1}^{n}, \ldots, \mathrm{K}_{4}^{n}$ of the graph $\bar{S}_{n}^{*}$. Consider the graph $\bar{S}_{1}^{*}$, then it is a bipartite graph on the torus. Fix a reference matching $M_{0}$ of $\bar{S}_{1}^{*}$. For every other perfect matching $M$ of $\bar{S}_{1}^{*}$, consider the superposition $M \cup M_{0}$ of $M$ and $M_{0}$, then $M \cup M_{0}$ consists of doubled edges and cycles. Let us define four parity classes for perfect matchings $M$ of $\bar{S}_{1}^{*}$ : (e,e) consists of perfect matchings $M$ for which cycles of 
$M \cup M_{0}$ circle the torus an even number of times horizontally and vertically; (e,o) consists of perfect matchings $M$, for which cycles of $M \cup M_{0}$ circle the torus an even number of times horizontally, and an odd number of times vertically; (o,e) and $(0,0)$ are defined in a similar way. By Tesler [17], one can construct an orientation of the edges of $\bar{S}_{1}^{*}$, so that the corresponding weighted adjacency matrix $\mathrm{K}_{1}^{1}$ has the following property: perfect matchings which belong to the same parity class have the same sign in the expansion of the determinant of $\mathrm{K}_{1}^{1}$. By an appropriate choice of sign, we can make the (e,e) class have the plus sign in $\operatorname{det} \mathrm{K}_{1}^{1}$, and the other three have minus sign. Consider a horizontal and a vertical cycle of $\bar{S}_{1}$. Then define $\mathrm{K}_{2}^{1}$ (resp. $\mathrm{K}_{3}^{1}$ ) to be the matrix $\mathrm{K}_{1}^{1}$ where the sign of the coefficients corresponding to edges crossing the horizontal (resp. vertical) cycle is reversed; and define $\mathrm{K}_{4}^{1}$ to be the matrix $\mathrm{K}_{1}^{1}$ where the sign of the coefficients corresponding to edges crossing both cycles are reversed.

The orientation of the edges of $\bar{S}_{1}^{*}$ defines a periodic orientation of the graph $S^{*}$. For every $n$, consider the graph $\bar{S}_{n}^{*}$ and the four matrices $\mathrm{K}_{1}^{n}, \mathrm{~K}_{2}^{n}, \mathrm{~K}_{3}^{n}, \mathrm{~K}_{4}^{n}$ defined as above. These matrices are called the Kasteleyn matrices of the graph $\bar{S}_{n}^{*}$.

The orientation defined on the edges of the graph $S^{*}$ is a clockwise odd orientation. Let $\mathrm{K}_{S}$ be the real Dirac operator indexed by the vertices of $S^{*}$ corresponding to this clockwise orientation, and let $K_{S}$ be the Dirac operator indexed by the vertices of $S^{*}$. Then Proposition 2 is a direct consequence of Lemmas 10, 11, 12 below.

\section{Lemma 10.}

$$
\lim _{n \rightarrow \infty} \mu_{n}^{S}\left(e_{1}, \ldots, e_{k}\right)=\left(\prod_{i=1}^{k} \mathrm{~K}_{S}\left(w_{i}, b_{i}\right)\right) \underset{1 \leq i, j \leq k}{\operatorname{det}} \mathrm{K}_{S}^{-1}\left(b_{i}, w_{j}\right) .
$$

Proof. The toroidal partition function $Z\left(\bar{S}_{n}^{*}, v\right)$ is defined to be the weighted sum (weighted by the function $v$ ) of dimer configurations of the graph $\bar{S}_{n}^{*}$. Then, by Tesler [17] (it is a generalization of a theorem of Kasteleyn [5]), we have

Theorem 6. $[5,17]$

$$
Z\left(\bar{S}_{n}^{*}, v\right)=\frac{1}{2}\left(-\operatorname{det} \mathrm{K}_{1}^{n}+\operatorname{det} \mathrm{K}_{2}^{n}+\operatorname{det} \mathrm{K}_{3}^{n}+\operatorname{det} \mathrm{K}_{4}^{n}\right) .
$$

Kenyon gives the following theorem for the Boltzmann measure $\mu_{n}^{S}$.

Theorem 7. [7] $\mu_{n}^{S}\left(e_{1}, \ldots, e_{k}\right)$ is equal to $\left(\prod_{i=1}^{k} \mathrm{~K}_{S}\left(w_{i}, b_{i}\right)\right)$ times

$$
\left(-\frac{\operatorname{det} \mathrm{K}_{1}^{n}}{2 Z\left(\bar{S}_{n}^{*}, v\right)} \operatorname{det}_{1 \leq i, j \leq k}\left(\left(\mathrm{~K}_{1}^{n}\right)^{-1}\left(b_{i}, w_{j}\right)\right)+\sum_{\ell=2}^{4} \frac{\operatorname{det} \mathrm{K}_{\ell}^{n}}{2 Z\left(\bar{S}_{n}^{*}, v\right)} \operatorname{det}_{1 \leq i, j \leq k}\left(\left(\mathrm{~K}_{\ell}^{n}\right)^{-1}\left(b_{i}, w_{j}\right)\right)\right) .
$$

This part of the argument can be found in [7]. Equation (10) is a weighted average of the four quantities $\operatorname{det}_{1 \leq i, j \leq k}\left(\left(\mathrm{~K}_{\ell}^{n}\right)^{-1}\left(b_{i}, w_{j}\right)\right)$, with weights $\frac{1}{2} \operatorname{det} \mathrm{K}_{\ell}^{n} / Z\left(\bar{S}_{n}^{*}, v\right)$. These weights are all in the interval $(-1,1)$ since, for every $\ell=1, \ldots, 4$, 
$2 Z\left(\bar{S}_{n}^{*}, v\right)>\left|\operatorname{det} \mathrm{K}_{\ell}^{n}\right|$. Indeed, $Z\left(\bar{S}_{n}^{*}, v\right)$ counts the weighted sum of dimer configurations of $\bar{S}_{n}^{*}$, whereas $\left|\operatorname{det} \mathrm{K}_{\ell}^{n}\right|$ counts some configurations with negative sign. Moreover, by Theorem 6 these weights sum to 1 , so that the weighted average converges to the same value as each $\operatorname{det}_{1 \leq i, j \leq k}\left(\left(\mathrm{~K}_{\ell}^{n}\right)^{-1}\left(b_{i}, w_{j}\right)\right)$.

Denote by $B_{S}$ (resp. $W_{S}$ ) the set of black (resp. white) vertices of $S^{*}$. Let us prove that for every $\ell=1, \ldots, 4$, and for every $w \in W_{S}, b \in B_{S},\left(\mathrm{~K}_{\ell}^{n}\right)^{-1}(b, w)$ converges to $\mathrm{K}_{S}^{-1}(b, w)$ on a subsequence of $n \mathrm{~s}$. The following theorem of [9] gives the convergence on a subsequence of $n \mathrm{~s}$ of the inverse Kasteleyn matrices of the graph $\bar{S}_{n}^{*}$.

Theorem 8. [9] For every $w \in W_{S}, b \in B_{S}, \ell=1, \ldots, 4$,

$$
\lim _{n \rightarrow \infty}^{\prime}\left(\mathrm{K}_{\ell}^{n}\right)^{-1}(b, w)=\frac{1}{(2 \pi)^{2}} \int_{S^{1} \times S^{1}} \frac{Q_{b, w}(z, u) u^{x} z^{y}}{P(z, u)} \frac{\mathrm{d} z}{z} \frac{\mathrm{d} u}{u},
$$

where $Q_{b, w}$ and $P$ are polynomials ( $Q_{b, w}$ only depends on the equivalence class of $w$ and $b$ ), and $x$ (resp. $y$ ) is the horizontal (resp. vertical) translation from the fundamental domain of $b$ to the fundamental domain of $w$.

Denote by $\mathrm{F}(b, w)$ the right hand side of (11). In [9], it is proved that $\mathrm{F}(b, w)$ converges to 0 as $|b-w| \rightarrow \infty$, as long as the dimer model is not in its frozen phase. Moreover, it is proved in [10] that dimer models on isoradial graphs are never in their frozen phase when the weight function is the critical one. Hence, we deduce that $\mathrm{F}(b, w)$ converges to 0 as $|b-w| \rightarrow \infty$.

Let us prove that for every $b \in B_{S}, w \in W_{S}, \mathrm{~F}(b, w)=\mathrm{K}_{S}^{-1}(b, w)$. Consider $w_{1}, w_{2} \in W_{S}$, and denote by $b_{1}, \ldots, b_{k}$ the neighbors of $w_{1}$. Assume $n$ is large enough so that the graph $\bar{S}_{n}^{*}$ contains $w_{1}, w_{2}, b_{1}, \ldots, b_{k}$, and so that the edges $w_{1} b_{j}$ do not cross the horizontal and vertical cycle of $\bar{S}_{n}$. Then, for every $\ell=1, \ldots, 4$,

$$
\sum_{b \in B_{S}} \mathrm{~K}_{\ell}^{n}\left(w_{1}, b\right)\left(\mathrm{K}_{\ell}^{n}\right)^{-1}\left(b, w_{2}\right)=\sum_{j=1}^{k} \mathrm{~K}_{\ell}^{n}\left(w_{1}, b_{j}\right)\left(\mathrm{K}_{\ell}^{n}\right)^{-1}\left(b_{j}, w_{2}\right)=\delta_{w_{1} w_{2}} .
$$

Moreover, $\mathrm{K}_{\ell}^{n}\left(w_{1}, b_{j}\right)=\mathrm{K}_{S}\left(w_{1}, b_{j}\right)$, so that taking the limit on a subsequence of $n \mathrm{~s}$, and using Theorem 8 , we obtain

$$
\sum_{j=1}^{k} \mathrm{~K}_{S}\left(w_{1}, b_{j}\right) \mathrm{F}\left(b_{j}, w_{2}\right)=\delta_{w_{1} w_{2}} .
$$

This is true for all $w_{1}, w_{2} \in W_{S}$. Moreover, $\lim _{|b-w| \rightarrow \infty} \mathrm{F}(b, w)=0$, so that by definition of the inverse real Dirac operator, and by the existence and uniqueness Lemma 9, we deduce that for all $b \in B_{S}, w \in W_{S}, \mathrm{~F}(b, w)=\mathrm{K}_{S}^{-1}(b, w)$.

Hence, $\mu_{n}^{S}\left(e_{1}, \ldots, e_{k}\right)$ converges to the right hand side of (9) on a subsequence of $n \mathrm{~s}$. By Sheffield's Theorem [16], this is the unique limit of the Boltzmann measures $\mu_{n}^{S}$, so that we have convergence for every $n$. 


\section{Lemma 11.}

$$
\left(\prod_{i=1}^{k} \mathrm{~K}_{S}\left(w_{i}, b_{i}\right)\right) \underset{1 \leq i, j \leq k}{\operatorname{det}}\left(\mathrm{K}_{S}^{-1}\left(b_{i}, w_{j}\right)\right)=\left(\prod_{i=1}^{k} K_{S}\left(w_{i}, b_{i}\right)\right) \underset{1 \leq i, j \leq k}{\operatorname{det}}\left(K_{S}^{-1}\left(b_{i}, w_{j}\right)\right) .
$$

Proof. By definition of the determinant, the left hand side of (12) is equal to

$$
\sum_{\sigma \in \mathcal{S}_{n}} \operatorname{sgn} \sigma\left(\prod_{i=1}^{k} \mathrm{~K}_{S}\left(w_{i}, b_{i}\right)\right) \mathrm{K}_{S}^{-1}\left(b_{1}, w_{\sigma(1)}\right) \ldots \mathrm{K}_{S}^{-1}\left(b_{k}, w_{\sigma(k)}\right),
$$

where $\mathcal{S}_{n}$ is the set of permutations of $n$ elements. A permutation $\sigma \in \mathcal{S}_{n}$ can be written as a product of disjoint cycles, so let us treat the case of each cycle separately. Refer to Sect. 4.2.2 for the definition of the function $\mathrm{f}_{w x}$.

- Suppose that in the product there is a 1-cycle, that is a point $j$ such that $\sigma(j)=j$. Then, using Remark 2 and Lemma 9, we obtain

$$
\begin{aligned}
\mathrm{K}_{S}\left(w_{j}, b_{j}\right) & =\mathrm{f}_{w_{j} b_{j}}(0) K_{S}\left(w_{j}, b_{j}\right), \\
\mathrm{K}_{S}^{-1}\left(b_{j}, w_{j}\right) & =\overline{\mathbf{f}_{w_{j} b_{j}}(0)} K_{S}^{-1}\left(b_{j}, w_{j}\right) .
\end{aligned}
$$

Moreover, $\overline{\mathfrak{f}_{w_{j} b_{j}}(0)}=\mathrm{f}_{w_{j} b_{j}}(0)^{-1}$, hence

$$
\mathrm{K}_{S}\left(w_{j}, b_{j}\right) \mathrm{K}_{S}^{-1}\left(b_{j}, w_{j}\right)=K_{S}\left(w_{j}, b_{j}\right) K_{S}^{-1}\left(b_{j}, w_{j}\right) .
$$

- Suppose that in the product there is an $\ell$-cycle, with $\ell \neq 1$. To simplify notations, let us assume $\sigma(1)=2, \ldots, \sigma(\ell)=1$, and let us prove the following (indices are written cyclically, i.e. $\ell+1 \equiv 1)$,

$$
\prod_{j=1}^{\ell} \mathrm{K}_{S}\left(w_{j}, b_{j}\right) \mathrm{K}_{S}^{-1}\left(b_{j}, w_{j+1}\right)=\prod_{j=1}^{\ell} K_{S}\left(w_{j}, b_{j}\right) K_{S}^{-1}\left(b_{j}, w_{j+1}\right) .
$$

Again, using Remark 2 and Lemma 9, we obtain

$$
\begin{aligned}
& \prod_{j=1}^{\ell} \mathrm{K}_{S}\left(w_{j}, b_{j}\right) \mathrm{K}_{S}^{-1}\left(b_{j}, w_{j+1}\right) \\
& \quad=\prod_{j=1}^{\ell} K_{S}\left(w_{j}, b_{j}\right) K_{S}^{-1}\left(b_{j}, w_{j+1}\right) \mathrm{f}_{w_{j} b_{j}}(0) \mathrm{f}_{w_{j+1} b_{j}}(0)^{-1} .
\end{aligned}
$$

Using the definition of the function $\mathrm{f}_{w x}$, and the fact that it is well defined yields

$$
\begin{aligned}
\prod_{j=1}^{\ell} \mathrm{f}_{w_{j} b_{j}}(0) \mathbf{f}_{w_{j+1} b_{j}}(0)^{-1} & =\prod_{j=1}^{\ell} \mathbf{f}_{w_{j} b_{j}}(0) \mathbf{f}_{b_{j} w_{j+1}}(0) \\
& =\prod_{j=1}^{\ell} \mathrm{f}_{w_{j} w_{j+1}}(0)=\mathbf{f}_{w_{1} w_{1}}(0)=1 .
\end{aligned}
$$


This proves Eq. (14). Combining Eqs. (13) and (14), and the fact that every permutation is a product of cycles, we obtain Lemma 11.

\section{Lemma 12.}

$\left(\prod_{i=1}^{k} K_{S}\left(w_{i}, b_{i}\right)\right) \underset{1 \leq i, j \leq k}{\operatorname{det}_{1}}\left(K_{S}^{-1}\left(b_{i}, w_{j}\right)\right)=\left(\prod_{i=1}^{k} K\left(w_{i}, b_{i}\right)\right) \underset{1 \leq i, j \leq k}{\operatorname{det}}\left(K^{-1}\left(b_{i}, w_{j}\right)\right)$.

Proof. Since $P$ is simply connected, for every $i, j=1, \ldots, k$, it contains a path of $\widetilde{R}$ from $w_{j}$ to $b_{i}$. Moreover by Theorem 3 , the coefficient of the inverse Dirac operator corresponding to $b_{i}, w_{j}$ only depends on such a path. Hence $K_{S}^{-1}\left(b_{i}, w_{j}\right)=$ $K^{-1}\left(b_{i}, w_{j}\right)$. We also have $\forall i=1, \ldots, k, K_{S}\left(w_{i}, b_{i}\right)=K\left(w_{i}, b_{i}\right)$, so that we deduce Lemma 12.

\subsection{Proof of Theorem 4}

The edges of the graph $R^{*}$ form a countable set. For every $i \in \mathbb{N}$, define $f_{i}$ : $\mathcal{M}\left(R^{*}\right) \rightarrow\{0,1\}$ by

$$
f_{i}(M)= \begin{cases}1 & \text { if the edge } e_{i} \text { belongs to } M \\ 0 & \text { else. }\end{cases}
$$

Fix $k \in \mathbb{N}$, and a $k$-tuple $\left(s_{1}, \ldots, s_{k}\right)$ of distinct elements of $\mathbb{N}$. Let $H \in \mathcal{B}\{0,1\}^{k}$, where $\mathcal{B}\{0,1\}^{k}$ denotes the Borel $\sigma$-field of $\{0,1\}^{k}$, and define a cylinder of rank $k$ by

$$
A_{\left(s_{1}, \ldots, s_{k}\right)}(H)=\left\{M \in \mathcal{M}\left(R^{*}\right) \mid\left(f_{s_{1}}(M), \ldots, f_{s_{k}}(M)\right) \in H\right\} .
$$

Then $A_{\left(s_{1}, \ldots, s_{k}\right)}(H)$ can be written as a disjoint union of cylinder sets,

$$
A_{\left(s_{1}, \ldots, s_{k}\right)}(H)=\bigcup_{i=1}^{m}\left\{e_{t_{i 1}}, \ldots, e_{t_{i \ell_{i}}}\right\},
$$

(recall that for every $i,\left\{e_{t_{i 1}}, \ldots, e_{t_{i \ell_{i}}}\right\}$ denotes the set of dimer configurations of $R^{*}$ containing the edges $\left.e_{t_{i 1}}, \ldots, e_{t_{i \ell_{i}}}\right)$. Define

$$
\mu_{\left(s_{1}, \ldots, s_{k}\right)}(H)=\sum_{i=1}^{m}\left(\left(\prod_{j=1}^{\ell_{i}} K\left(w_{t_{i j}}, b_{t_{i j}}\right)\right) \operatorname{det}_{1 \leq j, k \leq \ell_{i}}\left(K^{-1}\left(b_{t_{i j}}, w_{t_{i k}}\right)\right)\right) .
$$

Let $\mathrm{P}$ be a finite simply connected sub-graph of $\mathrm{R}$ such that, for every $i=$ $1, \ldots, m, P^{*}$ contains the edges $e_{t_{i 1}}, \ldots, e_{t_{i} \ell_{i}}$. Let $\mathrm{S}$ be a periodic rhombus tiling of the plane that contains $\mathrm{P}$ (given by Proposition 1). Then, by Proposition 2, $\mu_{\left(s_{1}, \ldots, s_{k}\right)}(H)=\lim _{n \rightarrow \infty} \mu_{n}^{S}\left(A_{\left(s_{1}, \ldots, s_{k}\right)}(H)\right)$. From this we deduce that for every $k$, and for every $k$-tuple $\left(s_{1}, \ldots, s_{k}\right), \mu_{\left(s_{1}, \ldots, s_{k}\right)}$ is a probability measure on $\mathcal{B}\{0,1\}^{k}$. Moreover, we deduce that the system of measures $\left\{\mu_{\left(s_{1}, \ldots, s_{k}\right)}:\left(s_{1}, \ldots, s_{k}\right)\right.$ is a 
$k$-tuple of distinct elements of $\mathbb{N}\}$ satisfy Kolmogorov's two consistency conditions. Applying Kolmogorov's extension theorem, we obtain the existence of a unique measure $\mu^{R}$, which satisfies (2).

Using the fact that the measure $\mu^{R}$ of a cylinder set is the limit of Boltzmann measures, we deduce that the measure $\mu^{R}$ is a Gibbs measure in the sense given in Sect. 1.

Assume moreover that the graph $R^{*}$ is doubly periodic. Then, for every cylinder set $\left\{e_{1}, \ldots, e_{k}\right\}$ of $R^{*}, \mu^{R}\left(e_{1}, \ldots, e_{k}\right)=\lim _{n \rightarrow \infty} \mu_{n}^{R}\left(e_{1}, \ldots, e_{k}\right)$. Moreover by [16] (see also [9]), the Boltzmann measures $\mu_{n}^{R}$ converge to the minimal free energy per fundamental domain Gibbs measure which is unique, so that this proves Theorem 4.

\section{Gibbs measure on the set of all triangular quadri-tilings}

Recall that $\mathcal{Q}$ is the set of all triangular quadri-tilings up to isometry, i.e. the set of all quadri-tilings whose underlying tiling is a lozenge tiling of the equilateral triangular lattice $\mathbb{T}$. Denote by $\mathcal{M}$ the set of dimer configurations corresponding to quadri-tilings of $\mathcal{Q}$. In this section, we first define the notion of Gibbs measure on $\mathcal{M}$; then we define a $\sigma$-algebra $\sigma(\mathcal{B})$ on $\mathcal{M}$, and give an explicit expression for a Gibbs measure $\mu$ on $(\mathcal{M}, \sigma(\mathcal{B}))$. We conjecture $\mu$ to be of minimal total free energy per fundamental domain among a four parameter family of ergodic Gibbs measures.

The notion of Gibbs measure on $\mathcal{Q}$ is a natural extension of the notion of Gibbs measure on dimer configurations of a fixed graph. Assume a weight function $v$ is assigned to quadri-tiles of triangular quadri-tilings of $\mathcal{Q}$, then a Gibbs measure on $\mathcal{Q}$ is a probability measure with the following property. If a triangular quadri-tiling is fixed in an annular region, then quadri-tilings inside and outside of the annulus are independent. Moreover, the probability of any interior triangular quadri-tiling is proportional to the product of the weights of the quadri-tiles. Using the bijection between $\mathcal{Q}$ and $\mathcal{M}$, this yields the definition of a Gibbs measure on $\mathcal{M}$.

Define $\mathcal{L}$ to be the set of lozenge-with-diagonals tilings of the plane, up to isometry. Define $\mathcal{L}^{*}$ to be the graph (which is not planar) obtained by superposing the dual graphs $L^{*}$ of lozenge-with-diagonals tilings $L \in \mathcal{L}$. Although some edges of $\mathcal{L}^{*}$ have length 0 , we think of them as edges of the one skeleton of the graph, so that to every edge of $\mathcal{L}^{*}$ there corresponds a unique quadri-tile in a lozengewith-diagonals tiling of $\mathcal{L}$. Let $e$ be an edge of $\mathcal{L}^{*}$, and let $q_{e}$ be the corresponding quadri-tile, then $q_{e}$ is made of two adjacent right triangles. If the two triangles share the hypotenuse edge, they belong to two adjacent lozenges; else if they share a leg, they belong to the same lozenge. Let us call these lozenge(s) the lozenge(s) associated to the edge $e$, and denote it/them by $\mathrm{I}_{e}$ (that is $\mathrm{I}_{e}$ consists of either one or two lozenges). Let $\mathrm{k}_{e}$ be the edge(s) of $\mathbb{T}^{*}$ corresponding to the lozenge(s) $\mathrm{I}_{e}$.

Let $e_{1}, \ldots, e_{k}$ be a subset of edges of $\mathcal{L}^{*}$, and define the cylinder set $\left\{e_{1}, \ldots, e_{k}\right\}$ of $\mathcal{L}^{*}$ to be the set of dimer configurations of $\mathcal{M}$ which contain these edges. Let us call connected cylinder any cylinder of $\mathcal{L}^{*}$ which has the property that the lozenge(s) associated to its edges form a connected path. Then every cylinder of $\mathcal{L}^{*}$ can be expressed as a disjoint union of connected cylinders. Consider $\mathcal{B}$ the field 
consisting of the empty set and of the finite disjoint unions of connected cylinders. Denote by $\sigma(\mathcal{B})$ the $\sigma$-field generated by $\mathcal{B}$.

Let $\mu^{\mathbb{T}}$ be the Gibbs measure on dimer configurations of the honeycomb lattice $\mathcal{M}\left(\mathbb{T}^{*}\right)$ given in [7]. As we have noted before, Theorem 4 is true for all doubly periodic isoradial graphs with critical weights on their edges. Then, the measure $\mu^{\mathbb{T}}$ coincides with the minimal free energy per fundamental domain Gibbs measure given by Theorem 4 , when the doubly periodic isoradial graph is the honeycomb lattice. As a corollary to Theorem 4, we obtain:

Corollary 1. There is a probability measure $\mu$ on $(\mathcal{M}, \sigma(B))$ such that for every connected cylinder $\left\{e_{1}, \ldots, e_{k}\right\}$ of $\mathcal{L}^{*}$,

$$
\mu\left(e_{1}, \ldots, e_{k}\right)=\mu^{L}\left(e_{1}, \ldots, e_{k}\right) \mu^{\mathbb{T}}\left(\mathrm{k}_{e_{1}}, \ldots, \mathrm{k}_{e_{k}}\right),
$$

where $L$ is the lozenge-with-diagonals tiling corresponding to any lozenge tiling $\mathrm{L}$ which contains the lozenges $\mathrm{I}_{e_{1}}, \ldots, \mathrm{I}_{e_{k}}$. Moreover $\mu$ is a Gibbs measure on $\mathcal{M}$, where the critical weight function is assigned to quadri-tiles.

Proof. Expression (15) is well defined, i.e. independent of the lozenge tiling L which contains the lozenges $\mathrm{I}_{e_{1}}, \ldots, \mathrm{I}_{e_{k}}$. Indeed, by definition of a connected cylinder set, the lozenges associated to the edges $e_{1}, \ldots, e_{k}$ form a connected path of lozenges, say $\gamma$. Let $L$ be a lozenge tiling that contains $\gamma$, and denote by $K_{L}$ the complex Dirac operator indexed by the vertices of the graph $L^{*}$. Then $K_{L}^{-1}\left(b_{i}, w_{j}\right)$ is independent of the lozenge tiling $\mathrm{L}$ which contains $\gamma$, indeed $K_{L}^{-1}\left(b_{i}, w_{j}\right)$ only depends on an edge-path of $\widetilde{R}$ from $w_{j}$ to $b_{i}$, and since $\mathrm{L}$ contains $\gamma$ which is connected, we can choose the edge-path to be the same for all such lozenge-withdiagonals tilings $L$. We then use the fact that $\mu^{\mathbb{T}}$ and $\mu^{L}$ are probability measures to prove the two conditions of Kolmogorov's extension theorem.

The measure $\mu$ is a Gibbs measure on $\mathcal{M}$ as a consequence of the fact that $\mu^{L}$ and $\mu^{\mathbb{T}}$ are Gibbs measure on $\mathcal{M}\left(L^{*}\right)$ and $\mathcal{M}\left(\mathbb{T}^{*}\right)$, respectively.

Assume that a weight function is assigned to quadri-tiles of quadri-tilings of $\mathcal{Q}$. Denote by $\Lambda$ the lattice which acts periodically on $\mathbb{T}$, and by $\mathbb{T}_{n}=\mathbb{T} / n \Lambda$, moreover suppose that $\mathbb{T}_{n}^{*}$ is bipartite (this is possible by eventually replacing $\Lambda$ by $2 \Lambda$ ). Define $\mathcal{Q}_{n}$ to be the set of triangular quadri-tilings whose underlying tiling is a lozenge tiling of $\mathbb{T}_{n}$. Denote by $\mu_{n}$ the Boltzmann measure on $\mathcal{Q}_{n}$; that is the probability of having a given subset of quadri-tiles in a quadri-tilings of $\mathcal{Q}_{n}$ chosen with respect to $\mu_{n}$ is proportional to the product of the weights of the quadri-tiles. We make the first following conjecture.

Conjecture 1. Suppose the critical weight function is assigned to quadri-tiles, then the Gibbs measure of Corollary 1 is the limit of the Boltzmann measures $\mu_{n}$.

Now fix $(s, t, p, q) \in \mathbb{R}^{4}$, and let $\mathcal{Q}_{n}^{(s, t, p, q)}$ be the subset of quadri-tilings of $\mathcal{Q}_{n}$ whose first height change is $(\lfloor n s\rfloor,\lfloor n t\rfloor)$ and second height change is $(\lfloor n p\rfloor,\lfloor n q\rfloor)$. Assuming that $\mathcal{Q}_{n}^{(s, t, p, q)}$ is non-empty, let $\mu_{n}^{(s, t, p, q)}$ be the conditional measure induced by $\mu_{n}$ on $\mathcal{Q}_{n}^{(s, t, p, q)}$. Denote by $Z_{n}^{(s, t, p, q)}$ the weighted sum of quadri-tilings 
of $\mathcal{Q}_{n}^{(s, t, p, q)}$, and define the total free energy per fundamental domain $\sigma(s, t, p, q)$ by:

$$
\sigma(s, t, p, q)=-\lim _{n \rightarrow \infty} \frac{1}{n^{2}} \log Z_{n}^{(s, t, p, q)}
$$

Then the following conjecture is inspired by a result of [16], see also [9].

Conjecture 2. For each $(s, t, p, q)$ for which $\mathcal{Q}_{n}^{(s, t, p, q)}$ is non-empty for $n$ sufficiently large, $\mu_{n}^{(s, t, p, q)}$ converges as $n \rightarrow \infty$ to an ergodic Gibbs measure $\mu^{(s, t, p, q)}$ of slope $(s, t, p, q)$. Furthermore $\mu_{n}$ itself converges to $\mu^{\left(s_{0}, t_{0}, p_{0}, q_{0}\right)}$ where $\left(s_{0}, t_{0}\right.$, $\left.p_{0}, q_{0}\right)$ is the limit of the slopes of $\mu_{n}$. If $\left(s_{0}, t_{0}, p_{0}, q_{0}\right)$ lies in the interior of the set $(s, t, p, q)$ for which $\mathcal{Q}_{n}^{(s, t, p, q)}$ is non-empty for $n$ sufficiently large, then every ergodic Gibbs measure of slope $(s, t, p, q)$ is of the form $\mu^{(s, t, p, q)}$ for some $(s, t, p, q)$ as above; that is $\mu^{(s, t, p, q)}$ is the unique ergodic Gibbs measure of slope $(s, t, p, q)$. Moreover, the measure $\mu^{\left(s_{0}, t_{0}, p_{0}, q_{0}\right)}$ is the unique one which has minimal total free energy per fundamental domain.

As a consequence, when the critical weight function is assigned to quadri-tiles, we conjecture the minimal total free energy per fundamental domain Gibbs measure to be given by the explicit expression of Corollary 1 .

\section{Asymptotics in the case of quadri-tilings}

Section 6.1 aims at giving a precise statement of Theorem 2 of Sect. 1 (see Theorem 9 below), and Sect. 6.2 gives consequences of Theorem 9 for the Gibbs measure $\mu^{R}$ of Theorem 4 and $\mu$ of Corollary 1 .

\subsection{Asymptotics of the inverse Dirac operator}

Refer to Fig. 11 for the following notations. Let $\ell_{1}^{\prime}, \ell_{2}^{\prime}$ be two disjoint side-length two rhombi in the plane, and let $\ell_{1}, \ell_{2}$ be the corresponding rhombi with-diagonals. Assume $\ell_{1}$ and $\ell_{2}$ have a fixed black and white bipartite coloring of their faces. Let $r_{1}$ and $r_{2}$ be the dual graphs of $\ell_{1}$ and $\ell_{2}$ ( $r_{1}$ and $r_{2}$ are rectangles), with the corresponding bipartite coloring of the vertices. Let $w$ be a white vertex of $r_{1}$, and $b$ a black vertex of $r_{2}$, then $w$ (resp. $b$ ) belongs to a boundary edge $e_{1}$ of $\ell_{1}$ (resp. $e_{2}$ of $\ell_{2}$ ). By Lemma 2, to the bipartite coloring of the faces of $\ell_{1}$ and $\ell_{2}$, there corresponds a bipartite coloring of the vertices of $\ell_{1}^{\prime}$ and $\ell_{2}^{\prime}$. Let $x_{1}$ (resp. $x_{2}$ ) be the black vertex of the edge $e_{1}$ (resp. $e_{2}$ ). Orient the edge $w x_{1}$ from $w$ to $x_{1}$, and let $\mathrm{e}^{i \theta_{1}}$ be the corresponding vector. Orient the edge $x_{2} b$ from $x_{2}$ to $b$, and let $\mathrm{e}^{i \theta_{2}}$ be the corresponding vector. Assume $\ell_{1}^{\prime}$ and $\ell_{2}^{\prime}$ belong to a rhombus tiling of the plane $\mathrm{R}$. Moreover, suppose that the bipartite coloring of the vertices of $R^{*}$ is compatible with the bipartite coloring of the vertices of $r_{1}$ and $r_{2}$.

Then we have the following asymptotics for the inverse Dirac operator $K^{-1}$ indexed by the vertices of $R^{*}$. Refer to Sect. 1 for comments about Theorem 9 . 

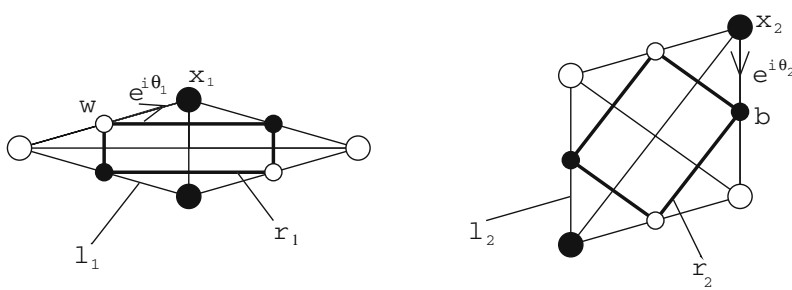

Fig. 11. Rhombi with diagonals $\ell_{1}, \ell_{2}$ and their dual graphs $r_{1}, r_{2}$

Theorem 9. As $|b-w| \rightarrow \infty, K^{-1}(b, w)$ is equal to

$$
\begin{aligned}
& \frac{1}{2 \pi}\left(\frac{1}{b-w}+\frac{\mathrm{e}^{-\mathrm{i}\left(\theta_{1}+\theta_{2}\right)}}{\bar{b}-\bar{w}}\right)+\frac{1}{2 \pi}\left(\frac{\mathrm{e}^{2 \mathrm{i} \theta_{1}}+\mathrm{e}^{2 \mathrm{i} \theta_{2}}}{(b-w)^{3}}+\frac{\mathrm{e}^{-\mathrm{i}\left(3 \theta_{1}+\theta_{2}\right)}+\mathrm{e}^{-\mathrm{i}\left(\theta_{1}+3 \theta_{2}\right)}}{(\bar{b}-\bar{w})^{3}}\right) \\
& \quad+O\left(\frac{1}{|b-w|^{3}}\right),
\end{aligned}
$$

where $\theta_{1}$ and $\theta_{2}$ are defined above.

Proof. Let us define an edge-path from $w$ to $b$ in $\widetilde{R}$ (the set of rhombi associated to the edges of $R^{*}$ ). Consider the bipartite coloring of the vertices of $\mathrm{R}$ (given by Lemma 2) associated to the bipartite coloring of the vertices of $R^{*}$. We define the graph $\mathrm{N}$ as follows. Vertices of $\mathrm{N}$ are black vertices of $R$, and two vertices of $N$ are connected by an edge if they belong to the same rhombus in $R$. The graph $N$ is connected because $R$ is. Each face of $N$ is inscribable in a circle of radius two. The circumcenter of a face of $N$ is the intersection of the rhombi in $R$, to which the edges on the boundary cycle of the face belong. Thus the circumcenter is in the closure of the face, and so faces of $\mathrm{N}$ are convex. Note that the vertices $x_{1}$ and $x_{2}$ are vertices of the graph $\mathrm{N}$.

Denote by $(x, y)$ the line segment from a vertex $x$ to a vertex $y$ of $\mathrm{N}$. An edge $u v$ of $\mathrm{N}$ is called a forward-edge for the segment $(x, y)$ if $\langle v-u, y-x\rangle \geq 0$. An edge-path $v_{1}, \ldots, v_{k}$ of $\mathrm{N}$ is called a forward-path for the segment $(x, y)$, if all the edges $v_{i} v_{i+1}$ are forward-edges for $(x, y)$. Similarly to what has been done in [8], let us define a forward-path of $\mathrm{N}$ for the segment $\left(x_{1}, x_{2}\right)$, from $x_{1}$ to $x_{2}$ (see Fig. 12). Let $F_{1}, \ldots, F_{\ell}$ be the faces of $\mathrm{N}$ whose interior intersect $\left(x_{1}, x_{2}\right)$ (if some edge of $\mathrm{N}$ lies exactly on $\left(x_{1}, x_{2}\right)$, perturb the segment $\left(x_{1}, x_{2}\right)$ slightly, using instead a segment $\left(x_{1}+\varepsilon_{1}, x_{2}+\varepsilon_{2}\right)$ for two generic infinitesimal translations $\left.\left(\varepsilon_{1}, \varepsilon_{2}\right)\right)$. Note that the number of such faces is finite because the rhombus tiling of the plane $\mathrm{R}$ has only finitely many different rhombi. Then for $j=1, \ldots, \ell-1, F_{j} \cap F_{j+1}$ is an edge $e_{j+1}$ of $\mathrm{N}$ crossing $\left(x_{1}, x_{2}\right)$. Set $v_{1}=x_{1}, v_{\ell}=x_{2}$, and for $j=1, \ldots, \ell-2$, define $v_{j+1}$ to be the vertex of $e_{j+1}$ such that the edge $e_{j+1}$ oriented towards $v_{j+1}$ is a forward-edge for $\left(x_{1}, x_{2}\right)$. Then, for $j=1, \ldots, \ell-1$, the vertices $v_{j}$ and $v_{j+1}$ belong to the face $F_{j}$. Take an edge-path from $v_{j}$ to $v_{j+1}$ on the boundary cycle of $F_{j}$, such that it is a forward-path for $\left(x_{1}, x_{2}\right)$. Such a path exists because faces of $\mathrm{N}$ are convex. Thus, we have built a forward-path of $\mathrm{N}$ for $\left(x_{1}, x_{2}\right)$, from $x_{1}$ to $x_{2}$. Denote by $u_{1}=x_{1}, u_{2}, \ldots, u_{k-1}, u_{k}=x_{2}$ the vertices of this path. 


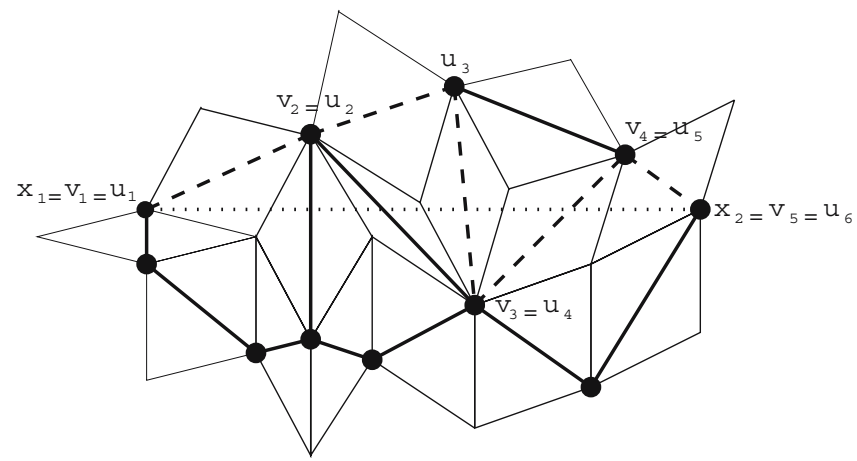

Fig. 12. Forward-path from $x_{1}$ to $x_{2}$ for the segment $\left(x_{1}, x_{2}\right)$

Let us now define an edge-path of $\widetilde{R}$ from $w$ to $b$. Note that the edges $w x_{1}$ and $x_{2} b$ are edges of $\widetilde{R}$. For $j=1, \ldots, k-1$, define the following edge-path of $\widetilde{R}$ from $u_{j}$ to $u_{j+1}$ (see Fig. 13). Remember that $u_{j} u_{j+1}$ is the diagonal of a rhombus of R, say $\tilde{\ell_{j}^{\prime}}$. Let $\tilde{r_{j}}$ be the dual graph of $\tilde{\ell_{j}}$. Let $u_{j}^{1}$ be the black vertex in $\tilde{r_{j}}$ adjacent to $u_{j}$, let $u_{j}^{2}$ be the crossing of the diagonals of $\tilde{\ell}_{j}$, and let $u_{j}^{3}$ be the white vertex in $\tilde{r_{j}}$ adjacent to $u_{j+1}$. Then the path $u_{j}, u_{j}^{1}, u_{j}^{2}, u_{j}^{3}, u_{j+1}$ is an edge-path of $\widetilde{R}$. Thus $w, x_{1}=u_{1}, u_{1}^{1}, u_{1}^{2}, u_{1}^{3}, u_{2}, \ldots, u_{k-1}, u_{k-1}^{1}, u_{k-1}^{2}, u_{k-1}^{3}, u_{k}=x_{2}, b$ is an edge-path of $\widetilde{R}$, from $w$ to $b$. Orient the edges in the path towards the black vertices of $R^{*}$, and away from the white vertices of $R^{*}$.

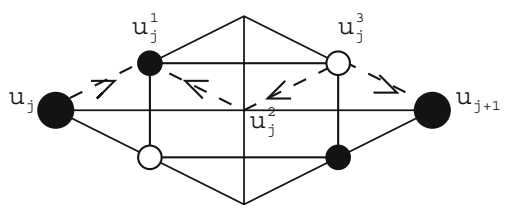

Fig. 13. Edge-path of $\widetilde{R}$ from $u_{j}$ to $u_{j+1}$

Let $\mathrm{e}^{i \beta_{j}^{1}}, \mathrm{e}^{i \beta_{j}^{2}}, \mathrm{e}^{i \alpha_{j}^{1}}, \mathrm{e}^{i \alpha_{j}^{2}}$ be the vectors corresponding, respectively, to the edges $u_{j} u_{j}^{1}, u_{j}^{3} u_{j+1}, u_{j}^{2} u_{j}^{1}, u_{j}^{3} u_{j}^{2}$. Without loss of generality, suppose that $x_{2}-x_{1}$ is real and positive. Then for $j=1, \ldots, k-1$, and $\ell=1,2$, we have:

$$
\cos \beta_{j}^{\ell}-\cos \alpha_{j}^{\ell}=\frac{\left\langle u_{j+1}-u_{j}, x_{2}-x_{1}\right\rangle}{2\left|x_{2}-x_{1}\right|} .
$$

Since $u_{1}, \ldots, u_{k}$ is a forward-path for $\left(x_{1}, x_{2}\right)$, this quantity is positive, thus $\cos \beta_{j}^{\ell} \geq \cos \alpha_{j}^{\ell}$.

Moreover, since there is only a finite number of different rhombi in $\mathrm{R}, k=$ $O(|b-w|)$. For the same reason, there is a finite number of angles $\beta_{j}^{\ell}$, and they are all in $[-\pi+\Delta, \pi-\Delta]$, for some small $\Delta>0$ (in the general case where the angle of the vector $x_{2}-x_{1}$ is $\theta_{0}$, the angles $\beta_{j}^{\ell}$ would be in the interval $\left[\theta_{0}-\pi+\Delta, \theta_{0}+\pi-\Delta\right]$ ). Thus by Theorem 4.3 of [8], we have that $K^{-1}(b, w)$ is equal to: 


$$
\frac{1}{2 \pi}\left(\frac{1}{b-w}+\frac{\gamma}{\bar{b}-\bar{w}}\right)+\frac{1}{2 \pi}\left(\frac{\xi_{2}}{(b-w)^{3}}+\frac{\gamma \overline{\xi_{2}}}{(\bar{b}-\bar{w})^{3}}\right)+O\left(\frac{1}{|b-w|^{3}}\right),
$$

where

and

$$
\gamma=\mathrm{e}^{-\mathrm{i}\left(\theta_{1}+\theta_{2}\right)} \prod_{j=1}^{k-1} \prod_{\ell=1}^{2} \mathrm{e}^{\mathrm{i}\left(-\beta_{j}^{\ell}+\alpha_{j}^{\ell}\right)}
$$

$$
\xi_{2}=\mathrm{e}^{2 \mathrm{i} \theta_{1}}+\mathrm{e}^{2 \mathrm{i} \theta_{2}}+\sum_{j=1}^{k-1} \sum_{\ell=1}^{2} \mathrm{e}^{2 \mathrm{i} \beta_{j}^{\ell}}-\mathrm{e}^{2 \mathrm{i} \alpha_{j}^{\ell}} .
$$

Note that for $j=1, \ldots, k-1$, we have $\alpha_{j}^{2} \equiv\left(\beta_{j}^{1}+\pi\right) \bmod [2 \pi]$, and $\beta_{j}^{2} \equiv\left(\alpha_{j}^{1}+\pi\right) \bmod [2 \pi]$, thus:

$$
\begin{aligned}
& \prod_{\ell=1}^{2} \mathrm{e}^{\mathrm{i}\left(-\beta_{j}^{\ell}+\alpha_{j}^{\ell}\right)}=\mathrm{e}^{\mathrm{i}\left(-\beta_{j}^{1}+\alpha_{j}^{1}\right)} \mathrm{e}^{\mathrm{i}\left(-\alpha_{j}^{1}-\pi+\beta_{j}^{1}+\pi\right)}=1, \\
& \sum_{\ell=1}^{2} \mathrm{e}^{2 i \beta_{j}^{\ell}}-\mathrm{e}^{2 \mathrm{i} \alpha_{j}^{\ell}}=\mathrm{e}^{2 \mathrm{i} \beta_{j}^{1}}-\mathrm{e}^{2 \mathrm{i} \alpha_{j}^{1}}+\mathrm{e}^{2 \mathrm{i}\left(\alpha_{j}^{1}+\pi\right)}-\mathrm{e}^{2 \mathrm{i}\left(\beta_{j}^{1}+\pi\right)}=0 .
\end{aligned}
$$

Therefore $\gamma=\mathrm{e}^{-\mathrm{i}\left(\theta_{1}+\theta_{2}\right)}, \xi_{2}=\mathrm{e}^{2 \mathrm{i} \theta_{1}}+\mathrm{e}^{2 \mathrm{i} \theta_{2}}$, which proves the theorem.

\subsection{Asymptotics of the Gibbs measures on quadri-tilings}

Let $\mathrm{R}$ be a rhombus tiling of the plane, and $R$ be the corresponding rhombus-withdiagonals tiling. Consider a subset of edges $e_{1}=w_{1} b_{1}, \ldots, e_{k}=w_{k} b_{k}$ of $R^{*}$, and recall that $\mu^{R}$ is the Gibbs measure on $\mathcal{M}\left(R^{*}\right)$ given by Theorem 4 .

Corollary 2. When $\forall j \neq i,\left|w_{j}-b_{i}\right| \rightarrow \infty$, then up to the second order term, $\mu^{R}\left(e_{1}, \ldots, e_{k}\right)$ only depends on the rhombi of $\mathrm{R}$ to which the vertices $b_{i}$ and $w_{j}$ belong, and else is independent of the structure of the graph $\mathrm{R}$.

Proof. This is a consequence of the explicit formula for $\mu^{R}\left(e_{1}, \ldots, e_{k}\right)$ of Theorem 4 , and of the asymptotic formula for the inverse Dirac operator of Theorem 9.

Recall that $\mathcal{L}^{*}$ is the non-planar graph obtained by superposing duals of lozengewith-diagonals tilings of $\mathcal{L}$. Let $e_{1}=w_{1} b_{1}, \ldots, e_{k}=w_{k} b_{k}$ be a subset of edges of $\mathcal{L}^{*}$. Define $\mathcal{L}^{E}$ to be the set of lozenge-with-diagonals tilings of the plane that contain the lozenges associated to the edges $e_{1}, \ldots, e_{k}$.

Corollary 3. When $\forall j \neq i,\left|w_{j}-b_{i}\right| \rightarrow \infty$, then up the second order term, $\mu^{L}\left(e_{1}, \ldots, e_{k}\right)$ is independent of $L \in \mathcal{L}^{E}$.

Proof. As in Sect. 5, we choose an embedding of $\mathcal{L}^{*}$ so that every edge of $\mathcal{L}^{*}$ uniquely determines the lozenge(s) it belongs to. Corollary 3 is then a restatement of Corollary 2. 
Recall that $\mu$ is the Gibbs on triangular quadri-tilings given by Corollary 1. Let $\mathrm{I}_{e_{1}}, \ldots, \mathrm{I}_{e_{k}}$ be the lozenges associated to the edges $e_{1}, \ldots, e_{k}$ of $\mathcal{L}^{*}$, and let $\mathrm{k}_{e_{1}}, \ldots, \mathrm{k}_{e_{k}}$ be the corresponding edges of $\mathbb{T}^{*}$.

Corollary 4. When $\forall j \neq i,\left|w_{j}-b_{i}\right| \rightarrow \infty$, and for every $L \in \mathcal{L}^{E}$, we have

$$
\mu\left(e_{1}, \ldots, e_{k}\right)=\mu^{L}\left(e_{1}, \ldots, e_{k}\right) \mu^{\mathbb{T}}\left(\mathrm{I}_{e_{1}}, \ldots, \mathrm{I}_{e_{k}}\right)+O\left(\frac{1}{(\bar{b}-\bar{w})^{3}}\right) .
$$

Proof. This is a consequence of the explicit formula for $\mu\left(e_{1}, \ldots, e_{k}\right)$ of Corollary 1 , and of Corollary 3.

Acknowledgements. We thank Richard Kenyon for proposing the quadri-tiling model and asking the questions related to it; we are grateful to him for the many enlightening discussions. We also thank the referee for the many pertinent remarks which have helped to increase the quality of this paper.

\section{References}

1. Billingsley, P.: Probability Theory. Wiley, New York, 1986

2. Cohn, H., Kenyon, R., Propp, J.: A variational principle for domino tilings. J Am Math Soc 14, 297-346 (2001)

3. Elkies, N., Kuperberg, G., Larsen, M., Propp, J.: Alternating sign matrices and domino tilings. J Algebraic Combin 1, 111-132 (1992)

4. Fisher, M., Temperley, H.: The dimer problem in statistical mechanics — an exact result. Philos Mag 6, 1061-1063 (1961)

5. Kasteleyn, P.W.: The statistics of dimers on a lattice. I. The number of dimer arrangements on a quadratic lattice. Physica 27, 1209-1225 (1961)

6. Kasteleyn, P.W.: Graph theory and crystal physics. Graph Theory and Theoretical Physics, pp. 43-110. London: Academic Press, 1967

7. Kenyon, R.: Local statistics of lattice dimers. Ann Inst H Poincaré Probab 33, 591-618 (1997)

8. Kenyon, R.: The Laplacian and Dirac operators on critical planar graphs. Invent Math 150, 409-439 (2002)

9. Kenyon, R., Okounkov, A., Sheffield, S.: Dimers and amoebas, math-ph/0311005. Ann Math (in press) (2006)

10. Kenyon, R., Okounkov, A.: Planar dimers and Harnack curves, math.AG/0311062. Duke Math J (in press) (2006)

11. Kenyon, R., Schlenker, J.-M.: Rhombic embeddings of planar graphs. Trans Am Math Soc 357(9), 3443-3458 (2005)

12. Kuperberg, G.: An exploration of the permanent-determinant method. Electron J Combin 5 (1998), Research Paper 46, 34 pp (electronic)

13. Mercat, C.: Exponentials form a basis of discrete holomorphic functions on a compact. Bull Soc Math Fr 132(2), 305-326 (2004)

14. Mercat, C.: Discrete period matrices and related topics, math-ph/0111043 (2001)

15. Propp, J.: Lattice structure for orientations of finite graphs, math.CO 0209005 (1994)

16. Sheffield, S.: PhD Thesis, Stanford University, 2003

17. Tesler, G.: Matchings in graphs on non-orientable surfaces. J Combin Theory Ser B 78(2), 198-231 (2000)

18. Thurston, W.P.: Conway’s tiling groups. Am Math Monthly 97, 757-773 (1990) 